\title{
COSMOLOGICAL CONSTRAINTS FROM THE SDSS MAXBCG CLUSTER CATALOG
}

\author{
Eduardo Rozo ${ }^{1}$, Risa H. Wechsler ${ }^{2}$, Eli S. Rykoff ${ }^{3}$, James T. Annis ${ }^{4}$, Matthew R. Becker ${ }^{5,6}$, August E. \\ Evrard $^{7,8,9}$, Joshua A. Frieman ${ }^{4,6,10}$ Sarah M. Hansen $^{11}$, Jiangang Hao ${ }^{7}$, David E. Johnston ${ }^{12}$, Benjamin P. \\ Koester $^{6,10}$, Timothy A. McKay ${ }^{7,8,9}$, Erin S. Sheldon ${ }^{13}$, DAvid H. Weinberg ${ }^{1,14}$ \\ Draft version February 21, 2009
}

\begin{abstract}
We use the abundance and weak lensing mass measurements of the SDSS maxBCG cluster catalog to simultaneously constrain cosmology and the richness-mass relation of the clusters. Assuming a flat $\Lambda \mathrm{CDM}$ cosmology, we find $\sigma_{8}\left(\Omega_{m} / 0.25\right)^{0.41}=0.832 \pm 0.033$ after marginalization over all systematics. In common with previous studies, our error budget is dominated by systematic uncertainties, the primary two being the absolute mass scale of the weak lensing masses of the maxBCG clusters, and uncertainty in the scatter of the richness-mass relation. Our constraints are fully consistent with the WMAP five-year data, and in a joint analysis we find $\sigma_{8}=0.807 \pm 0.020$ and $\Omega_{m}=0.265 \pm 0.016$, an improvement of nearly a factor of two relative to WMAP5 alone. Our results are also in excellent agreement with and comparable in precision to the latest cosmological constraints from X-ray cluster abundances. The remarkable consistency among these results demonstrates that cluster abundance constraints are not only tight but also robust, and highlight the power of optically-selected cluster samples to produce precision constraints on cosmological parameters.

Subject headings: cosmology: observation — cosmological parameters — galaxies: clusters — galaxies: halos
\end{abstract}

\section{INTRODUCTION}

The abundance of galaxy clusters has long been recognized as a powerful tool for constraining cosmological parameters. More specifically, from theoretical considerations (e.g. Press \& Schechter 1974; Bond et al. 1991; White et al. 1993; Sheth \& Tormen 2002) one expects the abundance of massive halos to be exponentially sensitive to the amplitude of matter fluctuations. Though some theoretical challenges remain (see e.g. Robertson et al. 2008; Stanek et al. 2009), this basic theoretical prediction has been confirmed many times in detailed numerical simulations, and a careful calibration of the abundance of halos as a function of mass for various cosmologies has been performed (see e.g. Jenkins et al. 2001; Warren

\footnotetext{
${ }^{1}$ Center for Cosmology and Astro-Particle Physics, The Ohio State University, Columbus, OH 43210 erozo@mps . ohio-state. edu

${ }^{2}$ Kavli Institute for Particle Astrophysics \& Cosmology, Department of Physics, and SLAC National Accelerator Laboratory Stanford University, Stanford, CA 94305

3 TABASGO Fellow, Physics Department, University of California at Santa Barbara, 2233B Broida Hall, Santa Barbara, CA 93106

${ }^{4}$ Fermi National Accelerator Laboratory, P.O. Box500, Batavia, IL 60510

${ }^{5}$ Department of Physics, The University of Chicago, Chicago, IL 60637

${ }^{6}$ Kavli Institute for Cosmological Physics, The University of Chicago, Chicago, IL 60637

${ }^{7}$ Physics Department, University of Michigan, Ann Arbor, MI 48109

8 Astronomy Department, University of Michigan, Ann Arbor, MI 48109

${ }_{9}$ Michigan Center for Theoretical Physics, Ann Arbor, MI 48109

10 Department of Astronomy and Astrophysics, The University of Chicago, Chicago, IL 60637

${ }^{11}$ University of California Observatories \& Department of Astronomy, University of California, Santa Cruz, CA 95064

12 Department of Physics \& Astronomy, Northwestern University, Evanston, IL 60208

13 Brookhaven National Laboratory, Upton, NY 11973

14 Department of Astronomy, The Ohio State University, Columbus, $\mathrm{OH} 43210$
}

et al. 2006; Tinker et al. 2008). Despite these successes, realizing the promise of cluster cosmology has proven difficult. Indeed, a review of observational results from the past several years yields a plethora of studies where typical uncertainties are estimated at the $\Delta \sigma_{8} \approx 0.05-0.10$ level despite a spread in central values that range from $\sigma_{8} \approx 0.65$ to $\sigma_{8} \approx 1.0$ (Viana \& Liddle 1996, 1999; Henry \& Arnaud 1991; Henry 2000; Pierpaoli et al. 2001; Borgani et al. 2001; Seljak 2002; Viana et al. 2002; Schuecker et al. 2003; Allen et al. 2003; Bahcall et al. 2003; Bahcall \& Bode 2003; Henry 2004; Voevodkin \& Vikhlinin 2004; Rozo et al. 2007b; Gladders et al. 2007; Rines et al. 2007).

The discrepancies among the various studies mentioned above is a manifestation of the fundamental problem confronting cluster abundance studies: theoretical predictions tell us how to compute the abundance of halos as a function of mass, but halo masses are not observable. Consequently, we are forced to rely on observable quantities such as X-ray temperature, weak lensing shear, or other such signals, to estimate cluster masses. This reliance on observable mass tracers introduces significant systematic uncertainties in the analysis; indeed, this is typically the dominant source of error (e.g. Henry et al. 2008).

There are two primary ways in which these difficulties can be addressed. One possibility is to reduce these systematic uncertainties through detailed follow-up observations of relatively few clusters, an approach exemplified in the work of Vikhlinin et al. (2008a). The second possibility is to use large cluster samples complemented with statistical properties of the clusters that are sensitive to mass to simultaneously fit for cosmology and the observable-mass relation of the cluster sample in question. Indeed, this is the basic idea behind the so called self-calibration approach, in which one uses the clustering of clusters (Schuecker et al. 2003; Estrada et al. 2008) and cluster abundance data to derive cosmological con- 
straints with no a-priori knowledge of the observablemass relation (Hu 2003; Majumdar \& Mohr 2004; Lima \& Hu 2004, 2005). There are, however, many other statistical observables that correlate well with mass, such as the cluster-shear correlation function (Sheldon et al. 2007), or even counts binned in multiple mass tracers (Cunha 2008). By including such data we can break the degeneracy between cosmology and the observablemass relation, thereby obtaining tight cosmological constraints while simultaneously fitting the observable-mass relation.

In this work, we derive cosmological constraints from the SDSS maxBCG cluster sample (Koester et al. 2007a) and the statistical weak lensing mass measurement from Johnston et al. (2007b). We then compare our result to three state-of-the-art cluster abundance studies of X-ray selected cluster samples (Mantz et al. 2008; Henry et al. 2008; Vikhlinin et al. 2008b) and demonstrate that our results are both consistent and competitive with these studies. This is the first time an optically selected catalog with masses estimated in a statistical way has produced constraints that are of comparable accuracy to the more traditional approach.

The paper is organized as follows. Section 2 presents the data used in our study. Section 3 describes our analysis, including the likelihood model and priors adopted in this work, and the way in which the analysis was implemented. Section 4 presents our main results, while sections 5.1, 5.2, and 5.3 discuss various sources of systematic uncertainties. Section 6.1 compares our results to the most recent results from X-ray selected cluster samples. Section 6.2 investigates the implications of our results for dark energy, and Section 6.3 discusses the prospects for improving our cosmological constraints from the maxBCG cluster sample in the future. Section 7 summarizes our main results and conclusions. Unless otherwise stated, all masses in this work are defined using an overdensity $\Delta=200$ relative to the mean matter density of the universe.

\section{DATA}

\subsection{MaxBCG Cluster Counts}

The maxBCG cluster catalog (Koester et al. 2007a) is an optically selected catalog drawn from 7,398 $\mathrm{deg}^{2}$ of DR4+ imaging data of the Sloan Digital Sky Survey (SDSS). ${ }^{15}$ The maxBCG algorithm exploits the tight $\mathrm{E} / \mathrm{S} 0$ ridgeline of galaxies in color-magnitude space to identify spatial overdensities of bright red galaxies. The tightness of the color distribution of cluster galaxies greatly suppresses the projection effects that have plagued optically selected cluster catalogs, and also allows for accurate photometric redshift estimates of the clusters $(\Delta z \approx 0.01)$. MaxBCG clusters are selected such that their photometric redshift estimates are in the range $z_{\text {photo }} \in[0.1,0.3]$, resulting in a nearly volume-limited catalog. A detailed discussion of the maxBCG cluster finding algorithm can be found in Koester et al. (2007b).

We bin the maxBCG cluster sample in nine richness bins spanning the range $N_{200} \in[11,120]$, corresponding roughly to $M \in\left[7 \times 10^{13} h^{-1} M_{\odot}, 1.2 \times 10^{15} h^{-1} M_{\odot}\right]$. Our richness measure $N_{200}$ is defined as the number of

15 We write DR4+ as the catalog used a few hundred degrees of imaging beyond those released with DR4.
TABLE 1

Abundance of MAXBCG Clusters

\begin{tabular}{cc}
\hline \hline Richness & No. of Clusters \\
\hline $11-14$ & 5167 \\
$14-18$ & 2387 \\
$19-23$ & 1504 \\
$24-29$ & 765 \\
$30-38$ & 533 \\
$39-48$ & 230 \\
$49-61$ & 134 \\
$62-78$ & 59 \\
$79-120$ & 31 \\
& \\
\hline
\end{tabular}

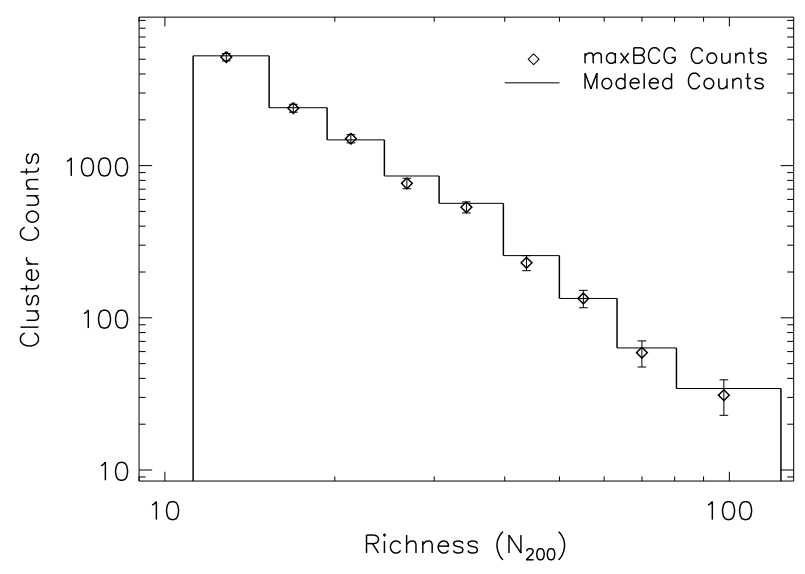

FIG. 1.- Observed (diamonds) and modeled (solid line) cluster counts as a function of richness in our best-fit model described in Section 4 . The model counts are computed using the best fit model detailed in Section 4, and are a good fit to the data.

red-sequence galaxies within a scaled radius such that the average galaxy overdensity interior to that radius is 200 times the mean galaxy density of the universe (see Koester et al. 2007a, for further details). The richness bins, and the number of clusters in each bin, are presented in Table 1. There are an additional five clusters with richness $N_{200}>120$. These five clusters have $N_{200}=126,139,156,164$, and 188, and are properly included in the analysis on an individual basis (see $§ 3.1$ for details).

Figure 1 shows the cluster counts corresponding to Table 1. Error bars between the various points are correlated. Also shown are the modeled counts from our bestfit model, detailed in Section 4. We show these model counts here for comparison purposes.

\subsection{MaxBCG Weak Lensing Masses}

Estimates of the mean mass of the maxBCG clusters as a function of richness are obtained through the weak lensing analysis described by Sheldon et al. (2007) and Johnston et al. (2007b). Briefly, Sheldon et al. (2007) binned the maxBCG cluster sample in richness bins as summarized in Table 2. Given a cluster in a specified richness bin, they use all cluster-galaxy pairs with the selected cluster as a lens to estimate the density contrast profile $\Delta \Sigma$ of the cluster. While these individual cluster profiles are very low signal-to-noise, averaging over all clusters within a richness bin allows one to obtain 
TABLE 2

Mean Mass of maxBCG Clusters

\begin{tabular}{ccc}
\hline \hline Richness & No. of Clusters & $\left\langle M_{200 b}\right\rangle\left[10^{14} M_{\odot}\right]$ \\
\hline $12-17$ & 5651 & 1.298 \\
$18-25$ & 2269 & 1.983 \\
$26-40$ & 1021 & 3.846 \\
$41-70$ & 353 & 5.475 \\
$71+$ & 55 & 13.03 \\
\hline
\end{tabular}

Note. - Masses listed here are based on those quoted in Johnston et al. (2007b), rescaled by the expected photometric redshift bias described in the text, and extrapolated to a matter overdensity $\Delta=200$ from the $\Delta=180$ value quoted in Johnston et al. (2007b). The masses have also been rescaled to the cosmology that maximizes our likelihood function, $\left(\sigma_{8}=0.80, \Omega_{m}=0.28\right)$.

accurate estimates for the mean density contrast profile of maxBCG clusters as a function of richness. The resulting profiles are fit using a halo model formalism to derive mean cluster masses by Johnston et al. (2007b). We then correct these masses upward by a factor of 1.18 due to the expected photometric redshift bias due to the dilution of the lensing signal from galaxies that are in front of the cluster lenses, but whose photometric redshift probability distribution extends past the cluster lens (see Mandelbaum et al. 2008b, for details). A very similar but independent analysis has also been carried out by Mandelbaum et al. (2008a), and we use the comparison between the two independent analysis to set the systematic error uncertainty of the weak lensing mass estimates (Rozo et al. 2008a). The final results of the weak lensing analysis summarized above are presented here in Table $2{ }^{16}$ Figure 2 shows the mean weak lensing masses from Table 2. Also shown are the mean masses computed using the best-fit model detailed in Section 4. The richness binning of the weak lensing mass estimates differs from that of the abundance data because of the larger number of clusters necessary within each richness bin to obtain high $\mathrm{S} / \mathrm{N}$ weak lensing measurements.

\section{ANALYSIS}

We employ a Bayesian approach for deriving cosmological constraints from the maxBCG cluster sample. We use only minimal priors placed on the parameters governing the richness-mass relation, relying instead on the cluster abundance and weak lensing data to simultaneously constrain cosmology and the richness-mass relation of the clusters. Details of the model, parameter priors, and implementation can be found below.

\subsection{Likelihood Model}

The observable vector $\mathbf{x}$ for our experiment is comprised of:

1. $N_{1}$ through $N_{9}$ : the number of clusters in each of the nine richness bins defined in Table 1 .

2. $(N \bar{M})_{1}$ through $(N \bar{M})_{5}$, the total mass contained in clusters in each of the five richness bins defined

\footnotetext{
16 The number of clusters in Table 2 is larger than that reported in Johnston et al. (2007b) due to masking in the weak lensing measurements. This additional masking does not bias the recovered masses in any way.
}

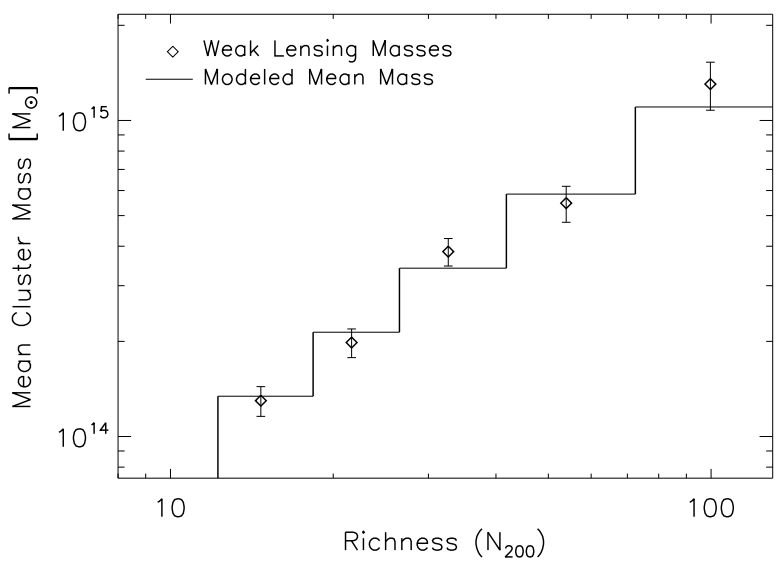

Fig. 2.- Mean weak lensing mass of maxBCG clusters as a function of richness. The diamonds with error bars correspond to our data, while the solid line shows the values predicted from our best-fit model (see Section 4 for details). We note the error bars are correlated, and the model is a good fit to the data.

in Table 2, computed assuming $\Omega_{m}=0.27$ and $h=0.71$. $^{17}$

We adopt a Gaussian likelihood model, which is fully specified by the mean and covariance matrix of our observables. Expressions for these quantities as a function of model parameters are specified below. We also multiply this Gaussian likelihood by a term that allows us to properly include the information contained in clusters with richness $N_{200}>120$. In this richness range clusters are very rare and a Gaussian likelihood model is not justified. Instead we adopt a likelihood model where the probability of having a cluster of a particular richness $N_{200}$ is binary (i.e. a Bernoulli distribution), with

$$
P\left(N \mid N_{200}\right)=\left\{\begin{array}{cl}
1-p & \text { if } N=0 \\
p & \text { if } N=1 .
\end{array}\right.
$$

Such a probability distribution is adequate so long as the probability of having two clusters of a given richness is infinitesimally small. Note that given this binary probability distribution, we have that the expectation value of the number of such clusters is simply $\left\langle N\left(N_{200}\right)\right\rangle=p$, and the likelihood is fully specified by the expectation value of our observable. We find that the likelihood of observing the particular richness distribution found for the maxBCG catalog for clusters of richness $N_{200} \geq 120$ is

$$
\mathcal{L}_{\text {tail }}=\prod_{N\left(N_{200}\right)=0}\left(1-\left\langle N\left(N_{200}\right)\right\rangle\right) \prod_{N\left(N_{200}\right)=1}\left\langle N\left(N_{200}\right)\right\rangle .
$$

The first product is over all richness $N_{200}>120$ and no clusters in them, and the second product is over richness bins which contain one cluster. The subscript tail reflects the fact that it is the likelihood of the tail of the abundance function. The final likelihood $\mathcal{L}=\mathcal{L}_{G} \mathcal{L}_{\text {tail }}$ is the product of the Gaussian likelihood $\mathcal{L}_{G}$ described earlier and the likelihood of the abundance function tail.

17 While Table 2 reports the masses after corrections, assuming $\Omega_{m}=0.28$, the actual input to our statistical analyis are the uncorrected masses from Johnston et al. (2007b), which assume $\Omega_{m}=0.27$. 
We note that the log-likelihood of the tail simplifies to

$$
\begin{aligned}
\ln \mathcal{L}_{\text {tail }}= & \sum_{N_{200}>120}\left\langle N\left(N_{200}\right)\right\rangle \\
& \quad-\sum_{N\left(N_{200}\right)=1}\left\langle N\left(N_{200}\right)\right\rangle+\ln \left\langle N\left(N_{200}\right)\right\rangle .
\end{aligned}
$$

An identical result is obtained assuming only Poisson variations in the number of clusters for $N_{200}>120$.

\subsection{Expectation Values}

To fully specify our likelihood model we need to derive expressions for the mean and variance of our observables. The model adopted in this work is very similar in spirit to that of Rozo et al. (2007b), so we present here only a brief overview of the formalism. Interested readers can find a detailed discussion in Rozo et al. (2007b).

We begin by considering the expected mean number of clusters in our sample. The number of halos within a redshift bin $z \in\left[z_{\min }, z_{\max }\right]$ and within a mass range $\left[M_{\min }, M_{\max }\right]$ is given by

$$
N=\int d M d z \frac{d n}{d M} \frac{d V}{d z} \psi(M) \phi(z)
$$

where $d n / d M$ is the halo mass function, $d V / d z$ is the comoving volume per unit redshift, and $\psi(M)$ and $\phi(z)$ are the mass and redshift binning functions. i.e. $\psi(M)=1$ if $M$ is within the mass bin of interest and zero otherwise, and $\phi(z)=1$ if $z$ is within the redshift bin of interest, but is zero otherwise.

In practice, we observe neither a cluster's mass nor its true redshift, but are forced to rely on the cluster richness $N_{200}$ as a mass tracer and to employ a photometric redshift estimate. Let then $P\left(N_{200} \mid M\right)$ denote the probability that a cluster of mass $M$ has a richness $N_{200}$, and let $P\left(z_{\text {photo }} \mid z\right)$ denote the probability that a cluster at redshift $z$ is assigned a photometric redshift $z_{\text {photo }}$. The binning function $\psi$ is now a function of richness rather than mass so $\psi\left(N_{200}\right)=1$ for $N_{200} \in\left[N_{200}^{\min }, N_{200}^{\max }\right]$. Likewise, the redshift binning function is now a function of photometric redshift $z_{\text {photo }}$. The total number of clusters in the maxBCG catalog becomes

$$
\langle N\rangle=\int d M d z \frac{d n}{d M} \frac{d V}{d z}\langle\psi \mid M\rangle\langle\phi \mid z\rangle,
$$

where

$$
\begin{aligned}
\langle\psi \mid M\rangle & =\int d N_{200} P\left(N_{200} \mid M\right) \psi\left(N_{200}\right) \\
\langle\phi \mid z\rangle & =\int d z_{\text {photo }} P\left(z_{\text {photo }} \mid z\right) \phi\left(z_{\text {photo }}\right) .
\end{aligned}
$$

The quantity $\langle\psi \mid M\rangle$ represents the probability that a halo of mass $M$ falls within the richness bin defined by $\psi$. We show these probabilities as a function of mass for each of the nine richness bins considered here in Figure 3. To make the figure, we have set all relevant model parameters to their best-fit value detailed in Section 4.

A similar argument allows us to write an expression for the expectation value for the total mass contained in clusters of a specified richness and redshift bin. This is given by

$$
\langle N \bar{M}\rangle=\int d M d z \frac{d n}{d M} \frac{d V}{d z} M\langle\psi \mid M\rangle\langle\phi \mid z\rangle .
$$

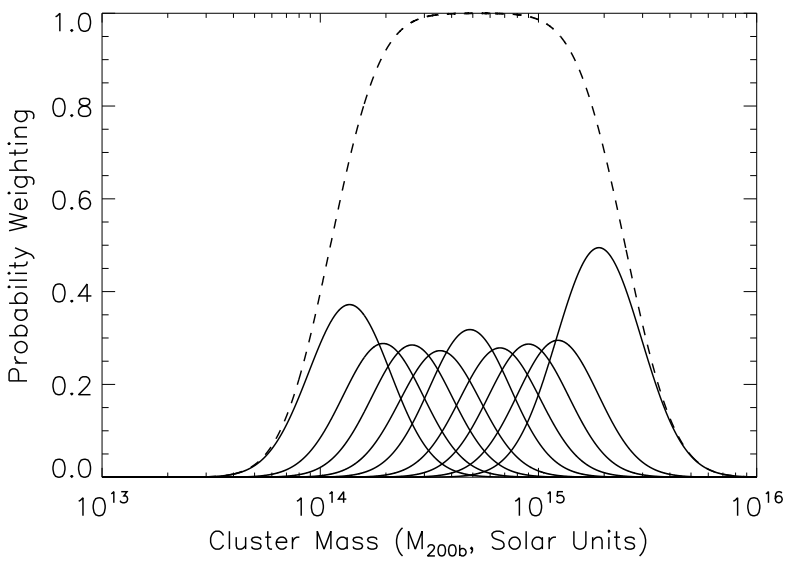

FIG. 3.- Mass selection function of the maxBCG algorithm. The nine solid curves represent the probability that a halo of the corresponding mass falls within each of the nine richness bins described in Table 1. The dashed line is the sum of all the binning functions, and is the probability that a halo of a given mass is assigned a richness $N_{200} \in[11,120]$, i.e. it is the mass selection function of the maxBCG algorithm over this richness range. These binning functions are all estimated using our best-fit model parameters, which are detailed in Section 4.

The notation $N \bar{M}$ reflects the fact that if $\bar{M}$ is the mean mass of the clusters of interest, the total mass contained in such clusters is $N \bar{M}$ where $N$ is the total number of clusters in said bin.

So far, our formulae adequately describe our experiment provided the weak lensing masses estimated by Johnston et al. (2007b) are fair estimates of the mean mass of the maxBCG clusters. In practice, there is an important systematic that needs to be properly incorporated in our analysis, and which slightly modifies our expression. We are referring to uncertainties in the photometric redshift estimates of the source galaxies employed in the weak lensing analysis. The main problem here is that the mean surface mass density profile $\Sigma(R)$ recovered by the weak lensing analysis is proportional to $1 /\left\langle\Sigma_{c}^{-1}\right\rangle$, the average inverse critical surface density of all lens-source pairs employed in the analysis. We introduce an additional weak lensing bias parameter $\beta$ such that if $\bar{M}_{\text {true }}$ is the true mean mass of a set of clusters, the weak lensing mass estimate $\bar{M}_{o b s}$ is given by $\bar{M}_{\text {obs }}=\beta \bar{M}_{\text {true }}$. Consequently, our final expression for the mean weak lensing masses of the maxBCG clusters is

$$
\langle N \bar{M}\rangle=\beta \int d M d z \frac{d n}{d M} \frac{d V}{d z} M\langle\psi \mid M\rangle\langle\phi \mid z\rangle .
$$

Priors on the parameter $\beta$ are discussed in Section 3.4.

\subsection{Covariance Matrix}

There are multiple sources of statistical uncertainty in the data. These include: (1) Poisson fluctuations in the number of halos of a given mass, (2) variance in the mean overdensity of the survey volume, and (3) fluctuations in the number of clusters at fixed richness due to stochasticity of the richness-mass relation. The covariance matrix of the observables is defined by the sum of the covariance matrices induced by each of the three sources of statistical fluctuation just mentioned. A detailed derivation of the relevant formulae is presented in Rozo et al. (2007a). 
Since this derivation generalizes trivially to include the mean mass as an additional observable - one needs only to introduce a mass weight in the formulae as appropriate — we will not repeat ourselves here.

There is, however, one additional source of statistical uncertainty that is not included in these calculation, namely measurement error in the weak lensing masses. More specifically, uncertainties in the recovered weak lensing masses is dominated by shape noise in the source galaxies. This error was estimated by Sheldon et al. (2007) using jackknife resampling, and was properly propagated into the computation of the weak lensing mass estimates by Johnston et al. (2007b). This error is added in quadrature to the diagonal elements of the covariance matrix corresponding to the mean mass measurements.

Finally, in addition to the errors summarized above, the covariance matrix is further modified due to systematic uncertainties in the purity and completeness of the sample. The basic set up is this: if $N_{\text {true }}\left(N_{200}\right)$ is the number of clusters one expects in the absence of systematics, and $N_{\text {obs }}\left(N_{200}\right)$ is the actual observed number of clusters, one has

$$
N_{\text {obs }}\left(N_{200}\right)=\lambda\left(N_{200}\right) N_{\text {true }}\left(N_{200}\right),
$$

where $\lambda$ is a factor close to unity that characterizes the purity and completeness systematics. If the sample is pure but incomplete, $\lambda$ is simply equal to the sample's completeness. For a complete but impure sample, $\lambda$ is one over the sample's purity. Note that, in general, $\lambda$ is itself a function of the cluster richness $N_{200}$. In Rozo et al. (2007b), we estimated the purity and completeness of the maxBCG cluster sample at $95 \%$ or higher for $N_{200} \geq 11$ (see Figures 3 and 6 in that paper), suggesting $\lambda=1.00 \pm 0.05$. Given that

$$
\operatorname{Var}\left(N_{\text {obs }}\right)=\operatorname{Var}(\lambda) N_{\text {true }}^{2}+\lambda^{2} \operatorname{Var}\left(N_{\text {true }}\right),
$$

it follows that we can incorporate the impact of this nuisance parameter by simply adding in quadrature the relative uncertainty introduced by $\lambda$ to the covariance matrix estimated in the previous section. A similar argument holds for the total mass contained in clusters within each richness bin. That is, if $\bar{M}_{\text {true }}$ is the true mean mass of clusters of richness $N_{200}$, and $\bar{M}_{o b s}$ is the observed mean mass, we expect

$$
(N \bar{M})_{o b s}=\tilde{\lambda}(N \bar{M})_{\text {true }},
$$

where $\tilde{\lambda}$ is a correction factor that accounts for the mass contribution of impurities in the sample. Unfortunately, it is impossible to know a priori what this factor $\tilde{\lambda}$ should be, even if we knew the correction factor $\lambda$ for cluster abundances. The reason is that false cluster detections will most certainly have a mass overdensity associated with them, just not that of a halo of the expected mass given the observed richness. Without a priori knowledge of this mass contribution, it is impossible to estimate the proper value of $\tilde{\lambda}$. In the extreme case that all false detections have mass $\bar{M}$, then the recovered value for $N \bar{M}$ will be biased by a factor $\tilde{\lambda}=\lambda$, which suggests adopting a fiducial value $\operatorname{Var}(\tilde{\lambda})=0.05^{2}$ to add to the diagonal matrix elements corresponding to the observed weak lensing masses. That is the approach we follow here. Throughout, we always set $\operatorname{Var}(\tilde{\lambda})=\operatorname{Var}(\lambda)$.

\subsection{Model Parameters and Priors}

Our analysis assumes a neutrino-less, flat $\Lambda$ CDM cosmology, and we fit for the values of $\sigma_{8}$ and $\Omega_{m}$. The Hubble parameter is held fixed at $h=0.7$, and the tilt of the primordial power spectrum is set to $n=0.96$ as per the latest WMAP results (Dunkley et al. 2008). The baryon density $\Omega_{b} h^{2}$ is also held fixed at its WMAP5 value $\Omega_{b} h^{2}=0.02273$. Of these secondary parameters, the two that are most important are the Hubble constant and tilt of the primordial matter power spectrum (Rozo et al. 2004). Section 5.2.1 demonstrates our results are robust to marginalization over these additional parameters.

The richness-mass relation $P\left(N_{200} \mid M\right)$ is assumed to be a log-normal of constant scatter. The mean logrichness at a given mass $\left\langle\ln N_{200} \mid M\right\rangle$ is assumed to vary linearly with mass, resulting in two free parameters. We comment on possible deviations from linearity in Section 5.3.1. For the two parameters specifying the mean richness-mass relation we have chosen the value of $\left\langle\ln N_{200} \mid M\right\rangle$ at $M=1.3 \times 10^{14} M_{\odot}$ and at $M=1.3 \times 10^{15} M_{\odot}$. These two are very nearly the values of the mean mass for our lowest and highest richness bins, and therefore roughly bracket the range of masses probed in our analysis. The value of $\left\langle\ln N_{200} \mid M\right\rangle$ at any other mass is computed through linear interpolation. We adopt flat priors on both of these parameters.

The scatter in the richness-mass relation $\sigma_{N_{200} \mid M}$ is defined as the standard deviation of $\ln N_{200}$ at fixed $M$, $\sigma_{N_{200} \mid M}^{2}=\operatorname{Var}\left(\ln N_{200} \mid M\right)$. We assume that this quantity is a constant that does not scale with mass, and adopt a flat prior $\sigma_{N_{200} \mid M} \in[0.1,1.5]$ for this parameter. We comment on possible deviations from constant scatter in Section 5.3.2. The minimum scatter allowed in our work $\left(\sigma_{N_{200} \mid M}=0.1\right)$ corresponds to a $10 \%$ scatter, which is the predicted scatter for $Y_{X}$ in simulations. $Y_{X}$ is usually regarded as the $\mathrm{X}$-ray mass tracer that is most tightly correlated with mass, so our prior on the scatter is simply the statement that richness estimates are less faithful mass tracers than $Y_{X}$.

We also place a prior on the converse scatter, that is, the scatter in mass at fixed richness $\sigma_{M \mid N_{200}}^{2}=$ $\operatorname{Var}\left(\ln M \mid N_{200}\right)$ at $N_{200}=40$. We emphasize that in our analysis the scatter $\sigma_{M \mid N_{200}}$ is considered an observable, not a parameter (the parameters is $\sigma_{N_{200} \mid M}$ ). The probability distribution $P\left(\sigma_{M \mid N_{200}}\right)$ is taken directly from the analysis by Rozo et al. (2008a), and can be roughly summarized as $\sigma_{M \mid N_{200}}=0.45 \pm 0.10(1 \sigma)$. This constraint is derived by demanding consistency between the observed $L_{X}-N_{200}$ relation of maxBCG clusters, the massrichness relation of maxBCG clusters derived from weak lensing, and the $L_{X}-M$ relation of clusters measured in the 400d survey (Vikhlinin et al. 2008b). To compute the observed scatter $\sigma_{M \mid N_{200}}$ as a function of our model parameters we directly compute the variance in log-mass for clusters in a richness bin $N_{200} \in[38,42]$. The variance in $\ln M$ due to the finite width of the bin is of order $(1 / 40)^{2} \approx 0.006$, which is to be compared to the intrinsic variance $\approx 0.45^{2} \approx 0.2$. Because the intrinsic variance is significantly larger than the variance due to using a finite bin width, our results are not sensitive to the width of the bin used in the implementation of the prior. We 
TABLE 3

\begin{tabular}{ccc}
\hline \hline Parameter $^{a}$ & Prior $^{b}$ & Importance $^{c}$ \\
\hline$\sigma_{8}$ & {$[0.4,1.2]$} & unrestrictive \\
$\Omega_{m}$ & {$[0.05,0.95]$} & unrestrictive \\
$\left\langle\ln N_{200} \mid M_{1}\right\rangle$ & flat & unrestrictive \\
$\left\langle\ln N_{200} \mid M_{2}\right\rangle$ & flat & unrestrictive \\
$\sigma_{N_{200} \mid M}$ & {$[0.1,1.5]$} & unrestrictive \\
$\beta$ & $1.00 \pm 0.06 ;[0.5,1.5]$ & restrictive \\
\hline$\sigma_{M \mid N_{200}}{ }^{d}$ & Rozo et al. (2008a) & restrictive \\
\hline
\end{tabular}

a The masses $M_{1}$ and $M_{2}$ are set to $1.3 \times 10^{14} M_{\odot}$ and $1.3 \times 10^{15} M_{\odot}$ respectively.

${ }^{\mathrm{b}}$ Priors of the form $[a, b]$ mean the parameter in question is restricted to values within that range (flat prior). Priors of the form $x=a \pm \delta a$ refer to a Gaussian prior of mean $\langle x\rangle=a$ and variance $\operatorname{Var}(x)=$ $(\delta a)^{2}$.

${ }^{\mathrm{c}}$ Column specifies whether our results are sensitive to the assumed priors. We refer to a prior as restrictive if our cosmological constraints are sensitive to the assumed prior, and unrestrictive otherwise. The only restrictive priors are that on the mass bias parameter $\beta$ and the prior on the scatter in mass at fixed richness.

${ }^{\mathrm{d}}$ Note $\sigma_{M \mid N_{200}}$ is not really a parameter in our analysis but an observable that can be computed given the six parameters above.

have explicitly checked that this is indeed the case. We have also checked that our results are insensitive to the location of the richness bin. That is, placing our prior on $\sigma_{M \mid N_{200}}$ at $N_{200}=30$ and $N_{200}=50$ gives results that are nearly identical to those obtained with our fiducial $N_{200}=40$ value. Finally, we note that in using the scatter measurement of Rozo et al. (2008a), who used an overdensity threshold of 500 relative to critical to define cluster masses, we are making the implicit assumption that the value of the current uncertainties in the scatter are much larger than any sensitivity to differences in the cluster mass definition. To address this concern, in Section 5.2.2 we discuss how the scatter prior impacts our results.

The redshift selection function $P\left(z_{\text {photo }} \mid z\right)$ is assumed to be Gaussian with $\left\langle z_{\text {photo }} \mid z\right\rangle=z$ and $\sigma\left(z_{\text {photo }} \mid z\right)=$ 0.008, as per the discussion in Koester et al. (2007a). We have explicitly checked that our results are not sensitive to our choice of parameters within the range $\delta\left\langle z_{\text {photo }} \mid z\right\rangle \approx 0.005$ and $\delta \sigma_{z_{\text {photo }} \mid z}=0.02$, which encompass the uncertainties in the photometric redshift distribution of the maxBCG clusters (Koester et al. 2007a).

Finally, we also adopt a prior on the weak lensing mass bias parameter, $\beta=1.0 \pm 0.06$, and allow it to vary over the range $[0.5,1.5]$. The width of our Gaussian prior is simply the mean difference between the Johnston et al. (2007b) masses (after correcting for photometric redshift bias) and those of Mandelbaum et al. (2008a) (for a more detailed discussion see Rozo et al. 2008a).

The total number of parameters that are allowed to vary in our Monte Carlo Markov Chain (MCMC) is six: $\sigma_{8}, \Omega_{m},\left\langle\ln N_{200} \mid M\right\rangle$ evaluated at $M=1.3 \times 10^{14} M_{\odot}$ and $M=1.3 \times 10^{15} M_{\odot}, \sigma_{N_{200} \mid M}$, and $\beta$. We summarize the relevant priors in Table 3 .

\subsection{Implementation}

We use the low baryon transfer functions of Eisenstein \& $\mathrm{Hu}$ (1999) to estimate the linear matter power spec- trum. The halo mass function is computed using Tinker et al. (2008). We use a mass definition corresponding to a 200 overdensity with respect to the mean matter density of the universe, and adopt the Sheth-Tormen expressions for the mass dependence of halo bias (Sheth \& Tormen 2002) (this enters into our analysis only in the calculation of sample variance). The likelihood function is sampled using a Monte Carlo Markov Chain (MCMC) approach with a burn in of 22,000 points during which the covariance matrix of the parameters is continually updated so as to provide an ideal sampling rate (Dunkley et al. 2005). We then run the chains for $10^{5}$ points, and use the resulting outputs to estimate the $68 \%$ and $95 \%$ likelihood contours in parameter space. For further details, we refer the reader to Rozo et al. (2007a).

The one point that is worth discussing here is our corrections for the dependence of the recovered weak lensing masses on the assumptions about cosmology used for the measurements. Johnston et al. (2007b) quote halo masses at an overdensity of 180 relative to the mean background of the universe. Given that we use a density contrast of 200 relative to mean in order to compute the halo mass function, we must re-scale the observed masses to our adopted mass definition. Moreover, the weak lensing analysis assumed $\Omega_{m}=0.27$. Given a different matter density parameter $\tilde{\Omega}_{m}$, the quoted mass will no longer correspond to an overdensity of 180 , but to an overdensity of $180\left(0.27 / \tilde{\Omega}_{m}\right)$. We explicitly apply this re-scaling to the observed weak lensing masses at each point in our MCMC. In practice, there is also an additional correction due to the dependence of the lensing critical surface density $\Sigma_{c}$ on the matter density parameter $\Omega_{m}$, as well as small corrections due to systematic variations in halo concentration with mass. However, these corrections are expected to be small, and are fully degenerate with the mass bias parameter $\beta$, so we do not include them here. The rescaling of the weak lensing masses is done using the fitting formulae in $\mathrm{Hu} \&$ Kravtsov (2003).

\section{RESULTS}

Figure 4 presents the $68 \%$ and $95 \%$ confidence regions for each pair of parameters in our fiducial analysis described in $\S 3$. Plots along the diagonal show the probability distributions of each quantity marginalized over the remaining parameters. Upper left plot showing the probability distribution of the mass parameter $\beta$ also shows the prior $\beta=1.00 \pm 0.06$ as a dashed curve. Our best fit model is summarized in Table 4, and is defined as the expectation value of all of our parameters. To test that our best fit model is a good model to the data, we performed $10^{4}$ Monte Carlo realizations of our best fit model, and evaluated the likelihood function for each of these realizations. Setting $\langle\ln \mathcal{L}\rangle=0$, from our Monte Carlo realizations we find $\ln \mathcal{L}=0.0 \pm 6.9$, which is to be compared to the data likelihood $\ln \mathcal{L}=-5.2$. The data likelihood is therefore consistent with our model, demonstrating the model is statistically a good fit.

In the discussion that follows, we restrict ourselves to the subset of plots which we find most interesting. Throughout, unless otherwise noted we summarize constraints on a parameter $p$ by writing $p=\bar{p}+\sigma_{p}$ where $\bar{p}$ and $\sigma_{p}$ are the mean and standard deviation of the likelihood distribution for $p$ marginalized over all other parameters. We use this convention even when the like- 


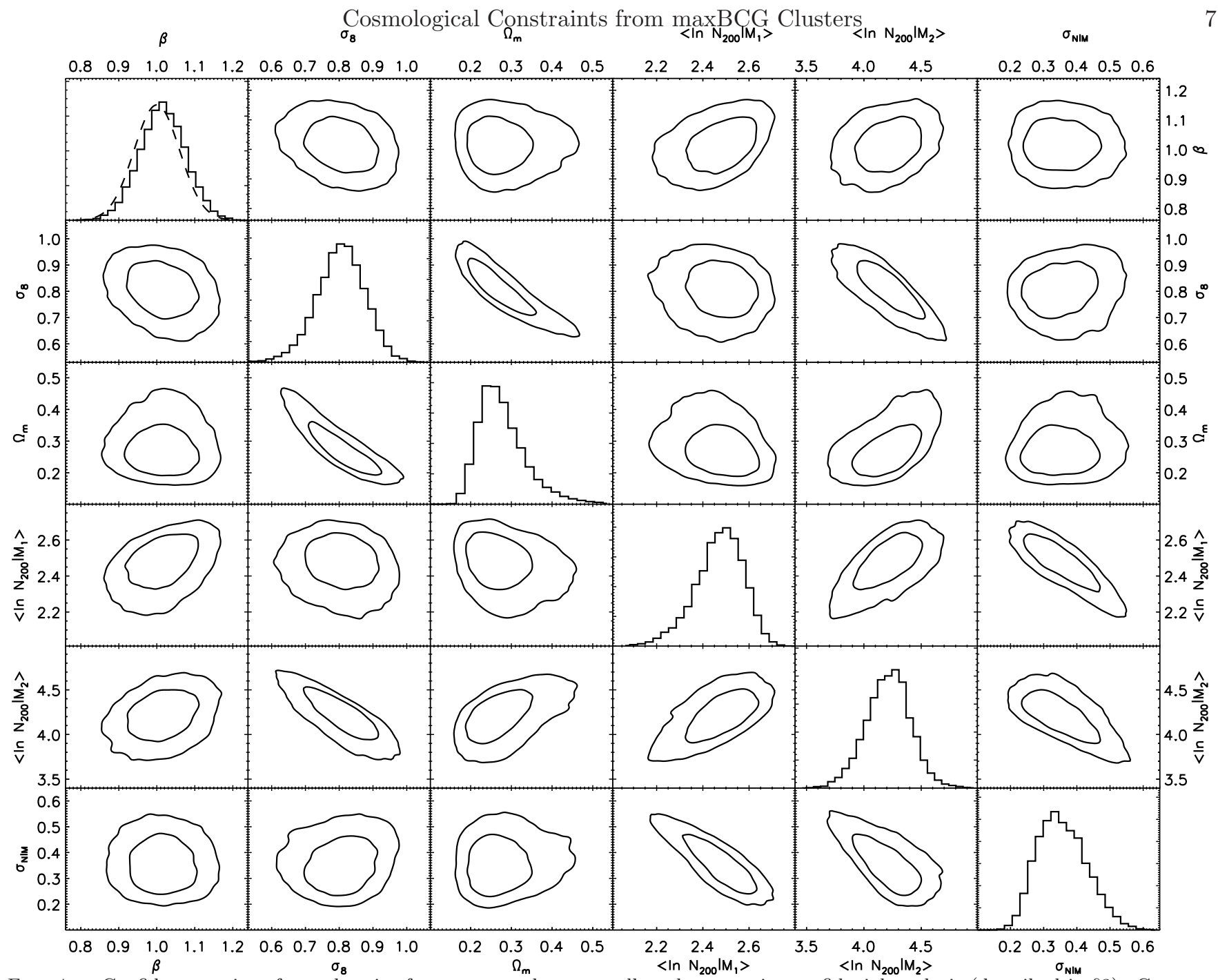

FIG. 4. - Confidence regions for each pair of parameters that were allowed to vary in our fiducial analysis (described in $\S 3$ ). Contours show $68 \%$ and $95 \%$ confidence regions. Plots along the diagonal show the probability distributions for each quantity marginalized over the remaining parameters. The probability distribution for the mass bias parameter $\beta$ also shows the prior $\beta=1.00 \pm 0.06$ assumed in the analysis.

TABLE 4

Best Fit Model

\begin{tabular}{ccc}
\hline \hline Parameter $^{a}$ & maxBCG & maxBCG+WMAP5 \\
\hline$\sigma_{8}$ & $0.804 \pm 0.073$ & $0.807 \pm 0.020$ \\
$\Omega_{m}$ & $0.281 \pm 0.066$ & $0.269 \pm 0.018$ \\
$\left\langle\ln N_{200} \mid M_{1}\right\rangle$ & $2.47 \pm 0.10$ & $2.48 \pm 0.10$ \\
$\left\langle\ln N_{200} \mid M_{2}\right\rangle$ & $4.21 \pm 0.19$ & $4.21 \pm 0.13$ \\
$\sigma_{N_{200} \mid M} \mid M$ & $0.357 \pm 0.073$ & $0.348 \pm 0.071$ \\
$\beta$ & $1.016 \pm 0.060$ & $1.013 \pm 0.059$ \\
\hline
\end{tabular}

aThe masses $M_{1}$ and $M_{2}$ are set to $1.3 \times 10^{14} M_{\odot}$ and $1.3 \times 10^{15} M_{\odot}$ respectively.

${ }^{\mathrm{b}}$ These values are obtained by including the WAMP5 prior $\sigma_{8}\left(\Omega_{m} / 0.25\right)^{-0.312}=0.790 \pm 0.024$. See Section 4.3 for details.

lihood function is obviously not Gaussian.

\subsection{Cosmological Constraints and Comparison to WMAP}

The solid curves in Figure 5 show the $68 \%$ and $95 \%$ confidence regions from our analysis. The "thin" axis of our error ellipse corresponds to $\sigma_{8}\left(\Omega_{m} / 0.25\right)^{0.41}=$
$0.832 \pm 0.033 .{ }^{18}$ The constraints on each of the individual parameters are $\sigma_{8}=0.80 \pm 0.07$ and $\Omega_{m}=$ $0.28 \pm 0.07$. The marginalized likelihood can be reasonably approximated by a log-normal distribution with $\ln \Omega_{m}=-1.313 \pm 0.183, \quad\left\langle\ln \sigma_{8}\right\rangle=-0.219 \pm 0.081$, and a correlation coefficient between $\ln \Omega_{m}$ and $\ln \sigma_{8}$ $r=-0.899$. Also shown in Figure 5 as dashed curves are the corresponding regions from the WMAP 5-year results (Dunkley et al. 2008). Our results are consistent with WMAP5. Combining the two experiments results in the inner filled ellipses, given by $\sigma_{8}=0.807 \pm 0.020$ and $\Omega_{m}=0.265 \pm 0.016$, with nearly no covariance between the two parameters $(r=0.008)$. These joint constraints on $\sigma_{8}$ and $\Omega_{m}$ represent nearly a factor of two improvement relative to the constraints from WMAP alone.

The shape of the confidence region is easy to interpret: since the number of massive clusters increases with both $\sigma_{8}$ and $\Omega_{m}$, in order to hold the cluster abundance fixed at its observed value any increase in $\sigma_{8}$ must be compensated by a decrease in $\Omega_{m}$, implying that a product of the form $\sigma_{8} \Omega_{m}^{\gamma}$ must be held fixed. The specific value of $\gamma$ de-

18 The exponent 0.41 is obtained by estimating the covariance matrix of $\ln \sigma_{8}$ and $\ln \Omega_{m}$, and finding the best constrained eigenvector. 


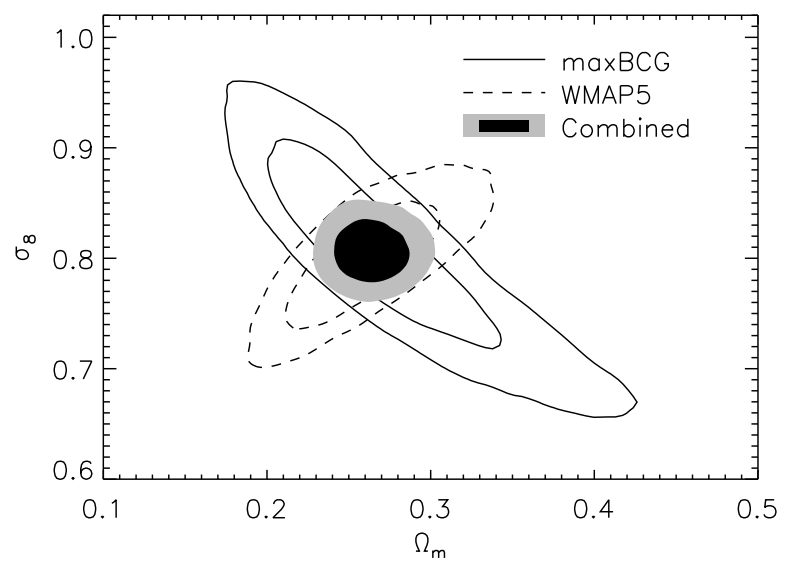

FIG. 5.- Constraints on the $\sigma_{8}-\Omega_{m}$ plane from maxBCG and WMAP 5 for a flat $\Lambda$ CDM cosmology. Contours show the $68 \%$ and 95\% confidence regions for maxBCG (solid), WMAP5 (dashed), and the combined results (filled ellipses). The thin axis of the maxBCG-only ellipse corresponds to $\sigma_{8}\left(\Omega_{m} / 0.25\right)^{0.41}=0.832 \pm$ 0.033. The joint constraints are $\sigma_{8}=0.807 \pm 0.020$ and $\Omega_{m}=$ $0.265 \pm 0.016$ (one-sigma errors).

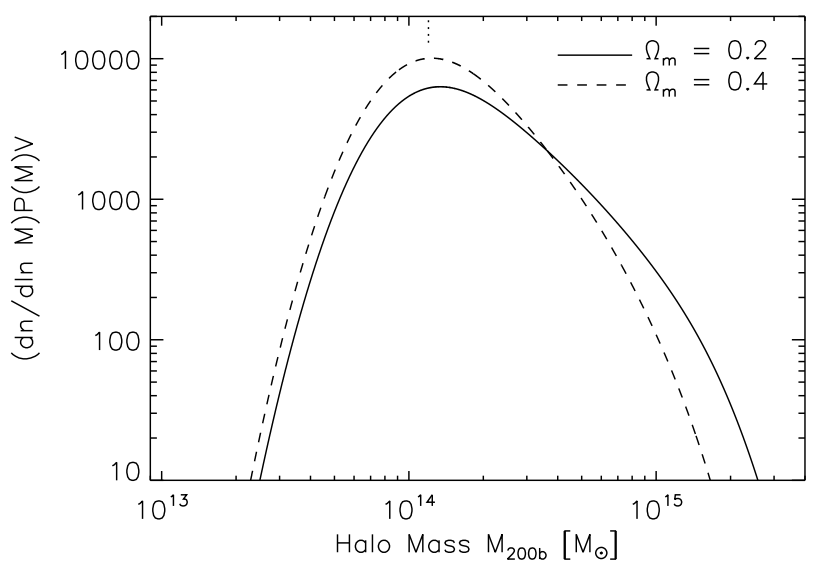

FiG. 6.- Halo mass function for two different cosmologies satisfying the maxBCG constraint $\sigma_{8}\left(\Omega_{m} / 0.25\right)^{0.41}=0.832$. The mass functions are weighted by the volume probed by the maxBCG catalog (computed assuming $\Omega_{m}=0.265$ ), and by the mass selection function shown in Figure 3. The maxBCG normalization condition $\sigma_{8}\left(\Omega_{m} / 0.25\right)^{0.41}=0.832$ results in a fixed halo abundance at a mass scale $M=3.6 \times 10^{14} M_{\odot}$. The dotted line at the top marks the mass scale at which the mean of the richness-mass relation is best constrained in our fiducial analysis.

pends on the mass scale that is best constrained from the data. The particular degeneracy recovered by our analysis corresponds to a mass scale $M=3.6 \times 10^{14} M_{\odot}$, which is about what we would expect (i.e. roughly half way between the lowest and highest masses probed by our data). Figure 6 illustrates this argument by showing the Tinker et al. (2008) halo mass function weighted by the mass selection function from Figure 3 for two different cosmologies: a low $\sigma_{8}$ (high $\Omega_{m}$ ) cosmology, and a high $\sigma_{8}$ (low $\Omega_{m}$ ) cosmology, where the product $\sigma_{8} \Omega_{m}^{0.41}$ has been held fixed to our best-fit value. We will refer back to Figure 6 multiple times in the following discussion.

\subsection{Constraints on the Richness-Mass Relation}

In our analysis, we parameterized the richness-mass relation in terms of its scatter, and the value of the mean $\left\langle\ln N_{200} \mid M\right\rangle$ at two mass scales, $M_{1}=1.3 \times 10^{14} M_{\odot}$ and $M_{2}=1.3 \times 10^{15} M_{\odot}$. We now re-parameterize this relation in terms of an amplitude and slope for $\left\langle\ln N_{200} \mid M\right\rangle$, selecting as the pivot point the mass scale at which the uncertainty in $\left\langle\ln N_{200} \mid M\right\rangle$ is minimized. We write then

$$
\left\langle\ln N_{200} \mid M\right\rangle=A+\alpha\left(\ln M-\ln M_{\text {pivot }}\right) .
$$

We find the error on the amplitude parameter is minimized for $M_{\text {pivot }}=1.09 \times 10^{14}$, which agrees well with the peak in the mass distribution of our clusters as shown in Figure 6. In what follows, we discuss only constraints on the richness-mass relation assuming this parameterization. A discussion of possible curvature in the richnessmass relation and/or mass scaling of its scatter is relegated to $§ 5.3$.

Figure 7 summarizes our constraints on the richnessmass relation after marginalizing over all other parameters. The best-fit values for each of the parameters are $A=2.34 \pm 0.10, \alpha=0.757 \pm 0.066$, and $\sigma_{N_{200} \mid M}=$ $0.357 \pm 0.073$. Note that for a pure power-law abundance function, one expects $\sigma_{N_{200} \mid M}=\alpha \sigma_{M \mid N_{200}}$, in accordance with our result.

Of these results, the constraints on the slope and scatter of the richness-mass relation are particularly worth noting. First, it is clear that the naive scaling $N_{200} \propto M$ is not satisfied, with the slope of the richness-mass relation being significantly smaller than unity. Second, the recovered scatter $\sigma_{N_{200} \mid M}=0.357 \pm 0.073$ is larger than the Poisson value $\sigma_{N_{200} \mid M} \approx 0.2$ that one might naively expect for clusters with $N_{200} \approx 30$ galaxies, which is the typical richness of clusters at the mass scale where mass function is best constrained.

Interpreting these results in terms of standard halo occupation model parameters requires care. The maxBCG richness is known to suffer from various sources of systematics including miscentering of clusters (Johnston et al. 2007a) and color off-sets in the richness estimates (Rozo et al. 2008b), both of which will impact the recovered richness-mass relation at some level. Moreover, any richness estimate will suffer to some extent from projection effects (Cohn et al. 2007), and discrepancies between assigned cluster radii and the standard mass-overdensity definitions used for halos. Disentangling the various contributions of each of these different sources of scatter to the total variance of the richness-mass relation is beyond the scope of this paper, and will not be considered further here.

Figure 7 also shows that the amplitude of the richnessmass relation is anti-correlated with the scatter. This is not surprising: at fixed cluster abundance, and given a fixed mass function, models with a high amplitude of the richness-mass relation result in halos that tend to be very rich. This means that the number of lower mass halos that scatter into higher richness must be low, or otherwise the abundance of clusters will be overpredicted. Consequently, high amplitude models must have low scatter, leading to an anti-correlation between the two parameters.

\subsection{Degeneracies Between Cosmology and the Richness-Mass Relation}




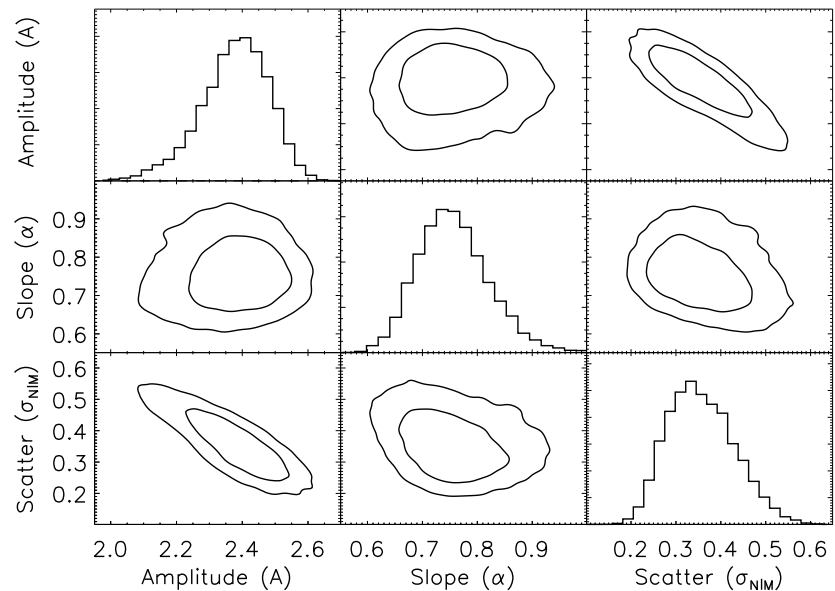

Fig. 7.- Constraints on parameters of the richness-mass relation. Countours indicate the $68 \%$ and $95 \%$ confidence regions; diagonal histograms indicate the probability distribution for each parameter, marginalized over the remaining parameters. The amplitude and slope parameters define the mean of the richness-mass relation as per Eq. 13. The pivot point of the relation occurs at $M=1.15 \times 10^{14} M_{\odot}$.

Figure 4 shows that the most significant correlation between cosmology and our fiducial richness-mass relation parameters is that between $\sigma_{8}$ and $\left\langle\ln N_{200} \mid M_{2}\right\rangle$ where $M_{2}$ is our higher reference mass $M_{2}=1.3 \times 10^{15} M_{\odot}$. Because the pivot point for the mean of the richnessmass relation is so close to our original low mass reference scale $M_{1}=1.3 \times 10^{14} M_{\odot}$ used to define $\left\langle\ln N_{200} \mid M\right\rangle$, it follows that $M_{2}$ must be closely related to $\alpha$, the slope of the richness-mass relation. We thus expect a strong degeneracy between $\sigma_{8}$ and $\alpha$ (see also Rozo et al. 2004).

Figure 8 shows that this is indeed the case. We can understand the origin of this anti-correlation by investigating Figure 6. We have seen that the data fixes the amplitude of the halo abundance at $M=3.9 \times 10^{14} M_{\odot}$. At the high mass end, however, the expected abundance of massive halos varies rapidly with $\sigma_{8}$. Low $\sigma_{8}$ models result in fewer massive halos, so high richness clusters will have relatively lower masses. That is, richness must increase steeply with mass, and hence $\alpha$ must be high, explaining the anti-correlation between $\sigma_{8}$ and $\alpha$.

Figure 8 also demonstrates how these constraints are improved when we include a WMAP five-year data prior $\sigma_{8}\left(\Omega_{m} / 0.25\right)^{-0.312}=0.790 \pm 0.024$. This prior corresponds to the error along the thin direction of the WMAP error ellipse. Since WMAP data breaks the $\sigma_{8}-\Omega_{m}$ degeneracy in the data, including the WMAP prior produces a tight constraint in the $\sigma_{8}-\alpha$ plane. The new marginalized uncertainty in the slope of the richnessmass relation is $\alpha=0.752 \pm 0.024$, significantly smaller than unity.

\section{SYSTEMATIC ERRORS}

We now consider the impact of three varieties of systematic errors on our analysis. Section 5.1 investigates observational systematics, Section 5.2 investigates systematics due to our assumed priors, and Section 5.3 investigates systematics due to the parameterization of the richness-mass relation.

\subsection{Observational Systematics}

In this section, we study how observational systematics affect the recovered cosmological constraints from our

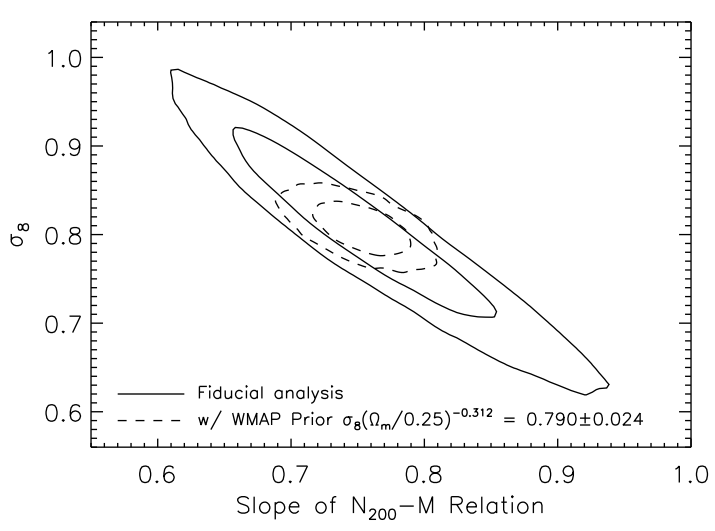

FIG. 8.- Confidence regions in the $\sigma_{8}-\alpha$ plane. Solid ellipses show the $68 \%$ and $95 \%$ likelihood regions. The tight correlation between $\sigma_{8}$ and $\alpha$, the slope of the richness-mass relatio, can be understood on the basis of Figure 6: a low $\sigma_{8}$ implies few massive halos, so to avoid under-predicting the abundance of rich clusters, galaxies must preferentially live in lower mass halos, resulting in a more rapidly rising richness-mass relation (i.e. higher slope). This degeneracy is broken upon inclusion of the WMAP five-year constraint $\sigma_{8}\left(\Omega_{m} / 0.25\right)^{-0.312}=0.790 \pm 0.024$ as an additional prior, as illustrated by the inner dashed ellipses in the Figure. The corresponding constraint on the slope of the richness-mass relation is $\alpha=0.752 \pm 0.024$.

analysis. We consider two such systematics: one, the impact of purity and completeness, and two, the impact of possible biases in the weak lensing mass estimates of the maxBCG clusters. We do not discuss uncertainties in the photometric redshifts for clusters at any length since, as discussed in Section 3.4, they are found to be negligible. This is not surprising, as the maxBCG photometric redshift estimates are extremely accurate $\left(\sigma_{z} \approx 0.008\right.$, Koester et al. 2007a).

\subsubsection{The Impact of Purity and Completeness}

Figure 9 compares the cosmological constraints obtained assuming perfect purity and completeness with those obtained assuming a $5 \%$ uncertainty in these quantities. While non-negligible, the $5 \%$ uncertainty in the completeness and purity function of the maxBCG cata$\log$ is far from the dominant source of uncertainty in our analysis. Moreover, this uncertainty elongates the error ellipse along its unconstrained direction, but has a minimal impact on the best constrained combination of $\sigma_{8}$ and $\Omega_{m}: \Delta \sigma_{8}\left(\Omega_{m} / 0.25\right)^{0.41}=0.033$ in our fiducial analysis, while $\Delta \sigma_{8}\left(\Omega_{m} / 0.25\right)^{0.41}=0.029$ assuming perfect purity and completeness, a mere $10 \%$ difference.

It is easy to understand why a $5 \%$ uncertainty in the purity and completeness has a minimal impact in our results. For $N_{200} \gtrsim 25$, the statistical uncertainties in the cluster abundances are larger than the $5 \%$ uncertainty in the counts from purity and completeness. Since the best constrained combination of cosmological parameters is driven primarily by high mass clusters, a $5 \%$ uncertainty in the purity and completeness functions has little impact on this parameter combination. How far the error ellipse extends along the degeneracy, however, is primarily driven by the observational constraints on the low end of the halo mass function (see Figure 6). Consequently, the $5 \%$ systematic uncertainty in the low richness cluster counts elongates the error ellipse along its major axis.

We conclude that for the expected level of purity and 


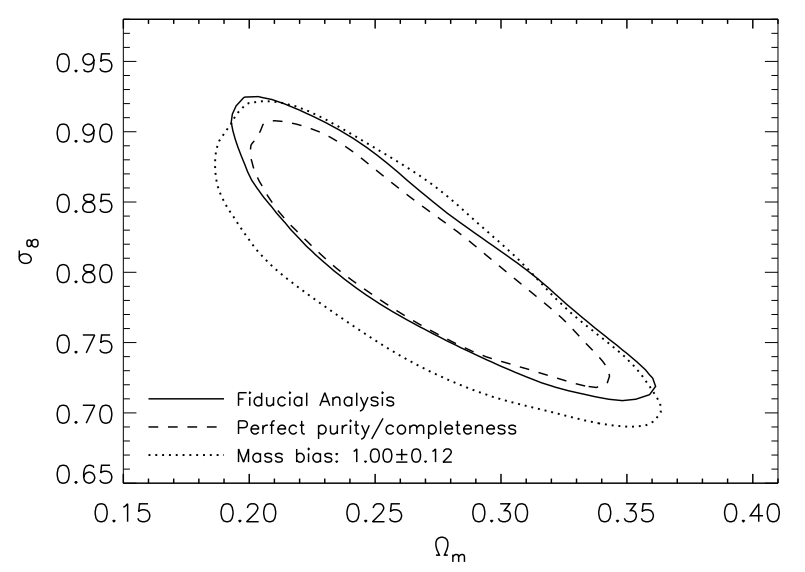

FIG. 9.- Effect of purity, completeness, and mass bias on parameter constraints. Plot shows $68 \%$ confidence regions assuming perfect purity and completeness (dashed), increasing the width of the weak lensing mass bias prior from $\beta=1.00 \pm 0.05$ to $\beta=1.00 \pm 0.12$ (dotted), and for our fiducial analysis (solid). We find the uncertainty in purity and completeness has a minimal impact on the best constrained combination of the $\sigma_{8}$ and $\Omega_{m}$ parameter, and therefore on the constraints from a joint maxBCG + WMAP5 analysis. The same is not true of the weak lensing mass bias parameter. Uncertainties in the maxBCG cluster masses are the dominant source of systematic in our current analysis, and increase the uncertainty of the parameter combination $\sigma_{8}\left(\Omega_{m} / 0.25\right)^{0.4}$ by $45 \%$ (see Figures 10 and 11 for comparison).

completeness of the maxBCG cluster sample, our cosmological constraints are robust to these systematics.

\subsubsection{Systematic Uncertainties of the Weak Lensing Mass Estimates}

In Section 2.2, we discussed that the weak lensing masses of Johnston et al. (2007b) were boosted by a factor of 1.18 to account for biases arising from scatter in the photometric redshift estimates (Mandelbaum et al. 2008b). Even with such a boost, the Johnston et al. (2007b) and the Mandelbaum et al. (2008a) mass estimates were not consistent, which led us in Section 3.2 to introduce a mass bias parameter $\beta$ that uniformly scales all masses by the same amount in order to account for any remaining biases. We now wish to explore how robust our results are to our estimate of this systematic uncertainty.

Figure 9 illustrates what happens if we repeat our fiducial analysis while doubling the width of the prior of $\beta$ from $\beta$ from $\beta=1.00 \pm 0.06$ to $\beta=1.00 \pm 0.12$. We find that the wider $\beta$ prior significantly increases the uncertainty in the parameter combination $\sigma_{8}\left(\Omega_{m} / 0.25\right)$ from $\Delta \sigma_{8}\left(\Omega_{m} / 0.25\right)^{0.41}=0.033$ to $\Delta \sigma_{8}\left(\Omega_{m} / 0.25\right)^{0.41}=$ 0.045 , corresponding to a $36 \%$ increase of the error bar. Using this new, wider prior, we find that the joint maxBCG + WMAP 5-year likelihood result in the cosmological constraints $\sigma_{8}=0.802 \pm 0.023$ and $\Omega_{m}=0.261 \pm 0.019$, which constitute $\mathrm{a} \approx 15 \%$ increase in the uncertainty of each of these parameters respectively. Even with this wider prior, however, adding the maxBCG constraint to the WMAP5 result improves the final cosmological constraints on $\sigma_{8}$ and $\Omega_{m}$ by a factor of 1.6 relative to those obtained using WMAP data alone.

We can understand the impact of the mass bias parameter on our cosmological constraints using Figure 6 .
A wider prior on $\beta$ implies that the mass scale of the maxBCG clusters is more uncertain, so the mass at which the cluster abundance is best constrained, i.e. the point at which the two curves in Figure 6 cross each other, is more uncertain. Consequently, the cluster normalization constraint $\sigma_{8} \Omega_{m}^{0.41}$ is weakened. The error along the long direction of the error ellipse does not change because the width of the mass range probed by the maxBCG clusters is largely independent of an overall mass bias.

One of the curious results that we have found in our study of the mass bias parameter $\beta$ is that the prior and posterior distributions of this parameter are different. In particular, we find that given the priors $\beta=1.00 \pm 0.06$ and $\beta=1.00 \pm 0.12$, the posterior distributions for $\beta$ are $\beta=1.02 \pm 0.06$ and $\beta=1.06 \pm 0.12$ respectively. Indeed, this explains why the error ellipse for our wider prior is displaced to the left of that of our fiducial analysis: the shift in $\beta$ corresponds to a change in the mass scale, which has to be compensated by a change in the matter density parameter $\Omega_{m}$.

We conclude that the uncertainty in the weak lensing mass estimates of the maxBCG clusters is an important source of systematic uncertainty in our analysis. In fact, it is the dominant source of systematic uncertainty in our analysis. We have explicitly considered the impact of photometric redshift estimates for source galaxies as the source of this uncertainty, but other biases to the lensing masses - for example if the fraction of miscentered clusters was over- or under-estimated by Johnston et al. (2007b) — would affect our results in a similar way.

\subsection{Prior-Driven Systematics}

Our analysis makes use of two important priors: that the only two cosmological parameters of interest are $\sigma_{8}$ and $\Omega_{m}$, and that the scatter in the richness-mass relation can be determined from X-ray studies as discussed in Rozo et al. (2008a). Here, we discuss how our results change if these priors are relaxed.

\subsubsection{Cosmological Priors}

After $\sigma_{8}$ and $\Omega_{m}$, cluster abundance studies are most sensitive to the Hubble parameter $h$ and the tilt $n$ of the primordial power spectrum. In Figure 10, we illustrate how the constraints on the $\sigma_{8}-\Omega_{m}$ plane are affected upon marginalization over $h$ and $n$ using Gaussian priors $h=0.7 \pm 0.1$ and $n=0.96 \pm 0.05$. As we can see, marginalizing over the Hubble parameter and the tilt of the power spectrum elongates the error ellipse, but it does not make it wider. Thus, the combination $\sigma_{8} \Omega_{m}^{0.41}$ remains tightly constrained, and a joint maxBCG and WMAP 5-year data analysis is robust to the details of the priors used for $h$ and $n$ when estimating the maxBCG likelihood function. We also investigated whether a non-zero neutrino mass could significantly affect our results. Using a prior $\sum m_{\nu}<1 \mathrm{eV}$, we find that massive neutrinos do not significantly affect our constrain on $\sigma_{8}\left(\Omega_{m} / 0.25\right)^{0.41}$. We conclude that holding the Hubble parameter and the tilt of the power spectrum fixed does not result in systematic uncertainties in the joint maxBCG + WMAP 5-year data analysis.

\subsubsection{The Impact of the Scatter Prior}

In Rozo et al. (2008a), we derived an empirical constraint on the scatter of the richness-mass relation by 


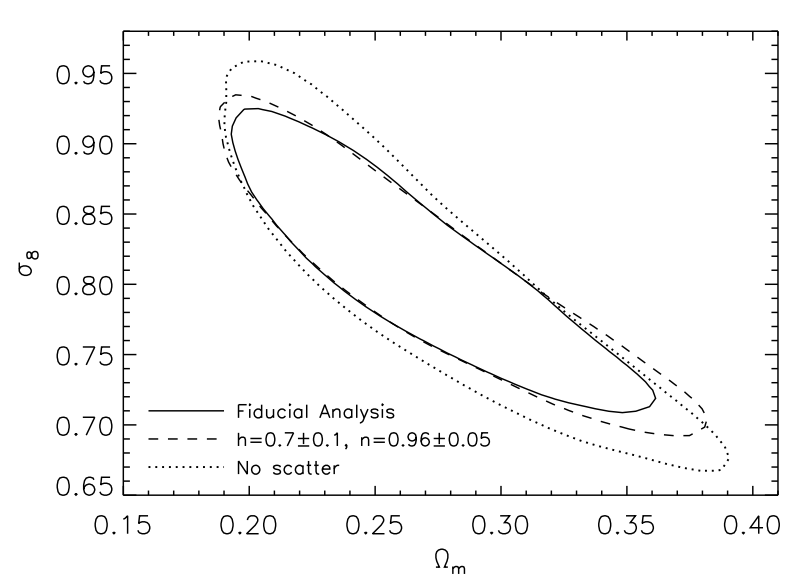

FIG. 10. - Effect of relaxing additional cosmological parameter priors on $\sigma_{8}$ and $\Omega_{m}$ constraints. Lines show $68 \%$ confidence regions for our fiducial analysis (solid), after marginalizing over Gaussian priors $h=0.7 \pm 0.1$ and $n=0.96 \pm 0.05$ (dashed), and using only a flat prior on the scatter in mass at fixed richness $\sigma_{M \mid N_{200}} \in[0.1,1.5]$ (dotted). We find that holding the Hubble parameter and power spectrum index fixed does not bias nor artificially tighten our constraint on $\sigma_{8} \Omega_{m}^{0.41}$. The scatter prior from Rozo et al. (2008a) on $\sigma_{M \mid N_{200}}$ employed in our fiducial analysis is found to have a significant impact on our data. More specifically, dropping this scatter prior increases the error along the short axis of our error ellipses by $36 \%$. We have also explored whether massive neutrinos significantly impact our constraint on $\sigma_{8} \Omega_{m}^{0.41}$, and find that for neutrino masses $\sum m_{\nu}<1 \mathrm{eV}$ there is no degradation of the error.

demanding consistency between X-ray, weak lensing, and cluster abundance data. The recovered scatter, however, characterized the richness-mass relation using a mass that was defined using an overdensity of 500 relative to the critical density of the universe. In this analysis, we use a density threshold of 200 relative to mean, so the use of the X-ray derived scatter prior is justified only if the scatter in the mass scaling between the two overdensity thresholds is not the dominant source of scatter. While we fully expect this assumption to hold, we have repeated our analysis without use of the scatter prior in order to cross-check our results.

Figure 10 summarizes our results. We find that our scatter prior tightens the error ellipse along both its short and long axis. This is as expected: without the scatter prior, the mass scale of the maxBCG clusters becomes less constrained, and consequently the halo mass function is less tightly constrained at all scales. The best constrained combination of $\sigma_{8}$ and $\Omega_{m}$ when dropping the Rozo et al. (2008a) prior on the scatter in the massrichness relation is $\sigma_{8}\left(\Omega_{m} / 0.25\right)^{0.48}=0.841 \pm 0.045$. This value represents a $36 \%$ increase in uncertainty relative to our fiducial analysis. The joint maxBCG + WMAP5 constraints in this case are $\sigma_{8}=0.805 \pm 0.021$ and $\Omega_{m}=0.264 \pm 0.017$.

Not surprisingly, prior knowledge of the scatter of the mass-richness relation can significantly enhance the constraining power of the maxBCG data set. Nevertheless, even without prior knowledge in the scatter the joint maxBCG+WMAP constraints improve upon the WMAP values by a factor of 1.7 .

\subsection{Parameterization Systematics}

One of the most important systematics that need to be addressed in studies where the observable-mass relation is parameterized in some simple way is how to assess the robustness of the results to changes in the parameterization of the observable-mass relation. Here, we have assumed that the richness-mass relation $P\left(N_{200} \mid M\right)$ is a log-normal of constant scatter and that $\left\langle\ln N_{200} \mid M\right\rangle$ varies linearly with $\ln M$. We now investigate how our results change if we relax some of these assumptions.

\subsubsection{Curvature in the Mean Richness-Mass Relation}

To investigate the impact of curvature in the mass richness relation, we assume $\left\langle\ln N_{200} \mid M\right\rangle$ is a piecewise linear function. We first specify $\left\langle\ln N_{200} \mid M\right\rangle$ at three mass scales $M_{1}, M_{2}$, and $M_{3}$, and define the value of $\left\langle\ln N_{200} \mid M\right\rangle$ at every other mass through linear interpolation in log-space. We set the minimum and maximum reference masses to the same values as before, $M_{1}=1.3 \times 10^{14} M_{\odot}$, and $M_{3}=1.3 \times 10^{14} M_{\odot}$. The intermediate reference mass is set to the geometric average of these two masses, $\ln M_{2}=0.5\left(\ln M_{1}+\ln M_{3}\right)$, or $M_{2}=3.66 \times 10^{14} M_{\odot}$. Note this mass scale is very nearly the same as the mass at which the halo mass function is best constrained.

Figure 11 shows how our cosmological constraints change with the introduction of mass dependence on the slope of the mean richness-mass relation $\left\langle\ln N_{200} \mid M\right\rangle$. We find that the thin axis of the error ellipse is not significantly affected by this more flexible parameterization, while the long axis of the error ellipse is somewhat lengthened. This is as expected: the high mass end of the halo mass function is only sensitive to how richness varies with mass for large $M$, and in this regime the more flexible parameterization does not introduce significantly more freedom. Thus, our data will tightly constrain the high mass end of the halo mass function just as well as did before, leading to no degradation in the error of $\sigma_{8} \Omega_{m}^{0.41}$. Once the high mass end of the richness-mass relation has been fixed, however, introducing curvature in $\left\langle\ln N_{200} \mid M\right\rangle$ dilutes the information contained in the low mass end of the halo mass function, thereby increasing the error ellipse along its long axis. Note the robustness of the $\sigma_{8} \Omega_{m}^{0.41}$ constraint also implies that the constraints of a joint maxBCG + WMAP5 analysis are not significantly affected by our choice of parameterization.

Irrespective of the impact our new parameterization of $\left\langle\ln N_{200} \mid M\right\rangle$ has on our cosmological constraints, it is fair to ask whether or not there is significant evidence for curvature of the mean richness-mass relation. Using a maximum likelihood ratio test, we find that the increase in likelihood due to curvature in the richnessmass relation is significant at the $50 \%$ level, less than $1 \sigma$. Thus, there is no evidence for curvature in the richnessmass relation. We have also explicitly confirmed that the slopes of the low and high mass end of the richness-mass relation are consistent with each other. Indeed, we find

$$
\left.\frac{d^{2}\left\langle\ln N_{200} \mid M\right\rangle}{d \ln M^{2}}\right|_{M=3.66 \times 10^{14} M_{\odot}}=0.05 \pm 0.07
$$

where we have assumed

$$
\frac{d^{2}\left\langle\ln N_{200} \mid M\right\rangle}{d \ln M^{2}}=\frac{f\left(M_{3}\right)+f\left(M_{1}\right)-2 f\left(M_{2}\right)}{0.5^{2}\left(\ln M_{3}-\ln M_{1}\right)^{2}} .
$$

and $f(M)=\left\langle\ln N_{200} \mid M\right\rangle$. 


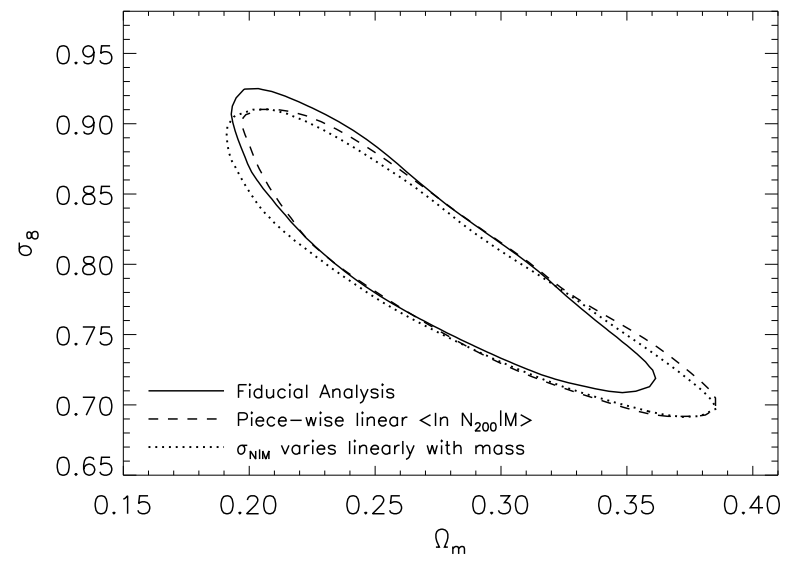

FIG. 11. - Effect of relaxing assumptions about the richnessmass relation on $\sigma_{8}-\Omega_{m}$ constraints. Contours show $68 \%$ confidence limits for our fiducial analysis (solid curve), assuming $\left\langle\ln N_{200} \mid M\right\rangle$ is a piece-wise linear function (dashed), and allowing $\sigma_{N_{200} \mid M}$ to vary linearly with mass (dotted). Giving additional freedom to the richness-mass relation has a minimal impact on our constraint on $\sigma_{8} \Omega_{m}^{0.41}$. Moreover, using a likelihood ratio test we find that there is no evidence in the data for curvature of the richness-mass relation, nor for a scatter that varies with mass. We conclude that our parameterization of $\left\langle\ln N_{200} \mid M\right\rangle$ and $\sigma_{N_{200} \mid M}$ do not introduce any significant systematics in our analysis.

\subsubsection{Scaling of the Scatter in the Richness-Mass Relation with Mass}

We now investigate whether allowing the scatter of the richness-mass relation to vary with mass has a significant impact on our cosmological parameters. For these purposes, we allow the scatter $\sigma_{N_{200} \mid M}$ to vary linearly with $\ln M$, and parameterize it by specifying its values at the reference masses $M_{1}=1.3 \times 10^{14} M_{\odot}$ and $M_{2}=1.3 \times 10^{15} M_{\odot}$. The value of $\sigma_{N_{200} \mid M}$ at any other mass is obtained through linear interpolation.

Figure 11 compares the cosmological constraints we obtain with our new model to those of our fiducial analysis with constant scatter. Once again, we find that the "thin" axis of the error ellipse is not significantly affected by the new more flexible parameterization, while the long axis is slightly elongated. The interpretation of these results is the same as those of $\$ 5.3 .1$. We have tested for evidence of scaling of the scatter in the richness-mass relation with halo mass using a likelihood ratio test. The increase in likelihood due to a linearly varying scatter is significant at the $39 \%$ level, implying there is no evidence of mass dependence in the scatter of the richness-mass relation in the data. We have also explicitly confirmed that the scatter at the low and high mass ends probed by the maxBCG cluster sample are consistent with each other. Indeed, our constraint on the slope of the mass dependence of the scatter in the richness-mass relation is

$$
\left.\frac{d \sigma_{N_{200} \mid M}}{d \ln M}\right|_{M=3.66 \times 10^{14} M_{\odot}}=0.00 \pm 0.06
$$

where we assumed

$$
\frac{d \sigma_{N_{200} \mid M}}{d \ln M}=\frac{\sigma_{N_{200} \mid M_{2}}-\sigma_{N_{200} \mid M_{1}}}{\ln M_{2}-\ln M_{1}} .
$$

We note the velocity dispersion analysis in Becker et al. (see 2007) points towards some mass dependence in the

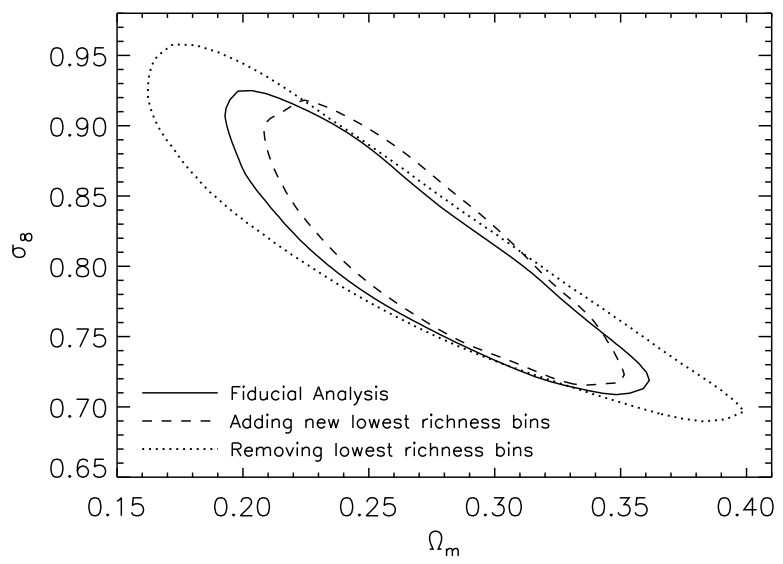

FIG. 12. - Effect of the minimum richness on $\sigma_{8}-\Omega_{m}$ constraints. Coutours show $68 \%$ confidence regions for our fiducial analysis (solid curve), an analysis where we include an additional richness bin, $\nu=9-10$, at the low end of the richness function (dashed), and an analysis where we drop the lowest richness bins considered in our fiducial analysis. Our cosmological constraints are consistent for all these analyses. We have also found that removing the most massive clusters from our analysis has minimal impact on our cosmological constraints.

scatter of the mass-richness relation, though part of this discrepancy is likely due to miscentering systematics (see Rozo et al. 2008a, for details). We are now in the process of reanalyzing the velocity dispersion data updating both our treatment of systematics, and substantially increasing the sample of spectroscopically sampled galaxies, so we defer a detailed discussion of these results to a future paper.

We conclude that our parameterization of the mean and scatter of the richness-mass relation does not introduce systematic errors in our analysis.

\subsubsection{Richness Range Considered}

We have tested whether there is cosmological information in the richness range $N_{200}>120$ by running MCMCs both with and without the contribution of these clusters to the likelihood function. We find that these two analyses yield nearly identical results. We have also explicitly confirmed that our results are robust to the lowest richness bin employed in the analysis. As we might expect, removing the lowest richness bin increases our uncertainties along the long axis of the error ellipse as shown in Figure 12. We also investigate adding a new lowest richness bin, consisting of clusters in with $N_{200}=$ 9-10, as well as the mean mass for clusters in the range $N_{200}=9-11$. This analysis rotates the error ellipse very slightly compared to our fiducial analysis, but does not significantly affect our results.

\section{DISCUSSION}

\subsection{Comparison to Other Work}

The main point of this section is to demonstrate two points:

1. The cosmological constraints from the maxBCG cluster catalog are competitive with the state of the art constraints derived from low redshift X-ray selected cluster samples. 
2. Despite the markedly different analyses and sources of systematic uncertainty, the cluster abundance constraints from the maxBCG cluster sample are in excellent agreement with those of X-ray selected samples. This demonstrates the robustness of cluster abundance studies as a tool of precision cosmology.

Given our goal, in this section we focus exclusively on the most recent cosmological constraints derived from low redshift X-ray cluster samples. In particular, we explicitly consider only three works: Mantz et al. (2008), who worked with the X-ray luminosity function, Henry et al. (2008), who worked with the X-ray temperature function, and Vikhlinin et al. (2008b), who estimated the low redshift halo mass function using the 400d X-ray survey (Burenin et al. 2007) with mass estimates based on $Y_{X}$ (Kravtsov et al. 2006). These three papers are the most recent analyses of X-ray selected cluster samples, and all recover tight cosmological constraints that are in excellent agreement with one another, while carefully accounting for the relevant systematics for each of their analyses.

Now, as we have discussed in previous sections, the main result from low redshift cluster abundance studies is a tight constraint on the value of $\sigma_{8} \Omega_{m}^{\gamma}$ where for maxBCG clusters $\gamma=0.41$. Other cluster samples, however, will have slightly different values of $\gamma$, which brings up the question of how can we fairly compare these various constraints. One way would be to simply quote the percent uncertainty in the relevant $\sigma_{8} \Omega_{m}^{\gamma}$ combination. However, we would like to have a clear graphical representation of this result. We have chosen to do this by plotting the $68 \%$ confidence regions of a simplified version of a joint cluster abundance + WMAP 5 analysis assuming a neutrino-less flat $\Lambda \mathrm{CDM}$ cosmology. We proceed as follows: given a cluster abundance experiment, we consider only the constraint on $\sigma_{8} \Omega_{m}^{\gamma}$, disregarding all other cosmological information. We then add a WMAP 5 prior $\sigma_{8}\left(\Omega_{m} / 0.25\right)^{-0.312}=0.790 \pm 0.024$, which corresponds to the thin axis of the error WMAP 5 error ellipse in the $\sigma_{8}-\Omega_{m}$ plane, and we compute the corresponding $68 \%$ confidence regions in the $\sigma_{8}-\Omega_{m}$ plane.

The result of this exercise is shown in Figure 13. The specific constraints from each of the works considered here are presented in Table 5. The agreement among the different analyses is excellent despite the tight error bars and the different sources of systematic uncertainties. This agreement clearly demonstrates not only that optically selected cluster samples can produce cosmological constraints that are competitive with those of X-ray selected cluster samples, but also that systematic uncertainties have been properly estimated.

\subsection{Low Redshift Cluster Abundances and The Equation of State of Dark Energy}

Detailed analyses exploring how cluster abundances help improve dark energy constraints have been presented by previous groups, most recently by Mantz et al. (2008) and Vikhlinin et al. (2008b). Rather than duplicating their work, in this section we opt for performing a simple analysis that captures the essential physics behind the Mantz et al. (2008) and Vikhlinin et al. (2008b) results, which helps illustrate exactly why and how clusters

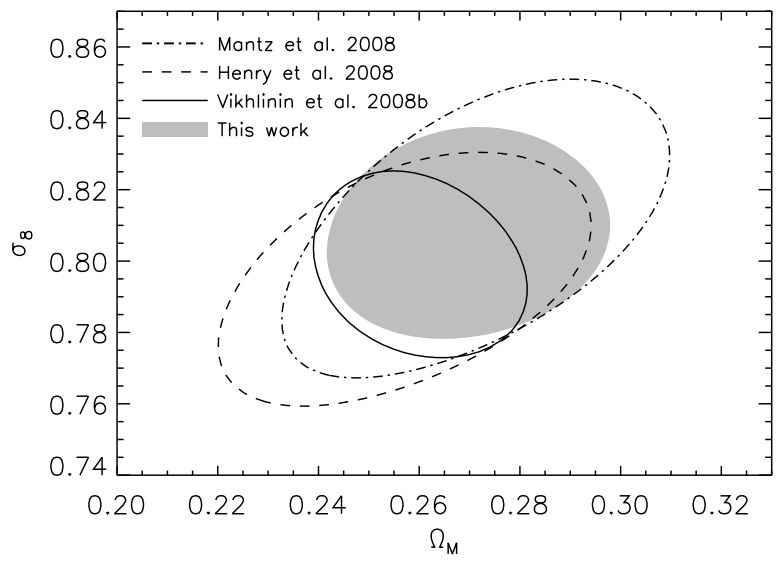

Fig. 13.- Comparion of optical and X-ray cluster abundance constraints on $\sigma_{8}-\Omega_{m}$. Contours show $68 \%$ confidence regions for a joint WMAP5 and cluster abundance analysis assuming a flat $\Lambda \mathrm{CDM}$ cosmology. In addition to our results (filled ellipse), we consider the latest cluster abundance constraints from the low redshift cluster luminosity function (dashed Mantz et al. 2008), temperature function (dash-dot Henry et al. 2008), and mass function as estimated with $Y_{X}$ (solid Vikhlinin et al. 2008b). All four studies are in excellent agreement with each other despite the tight cosmological constraints and the different sources of systematic uncertainty among the various analyses.

complement $\mathrm{CMB}$, supernova, and BAO studies.

We begin by focusing on the somewhat surprising result by Vikhlinin et al. (2008b) that a joint WMAP5 and low redshift cluster abundance experiment does not produce an interesting constraint on the equation of state of dark energy $w$. The reason this is surprising is that WMAP5 has measured the amplitude of the power spectrum at recombination to high accuracy. Given this value and a cosmological model, one can predict the value of $\sigma_{8}$ today. By demanding that this prediction agrees with the cluster normalization condition, one ought to obtain a tight constraint on the dark energy equation of state.

To understand why this is not the case, consider first the WMAP5 results. The parameters $w$ and $\Omega_{m}$ are strongly degenerate given the WMAP5 data alone, as shown in Figure 14. The value of $\sigma_{8}$ implied by the WMAP5 data depends sensitively on these two parameters, so a large uncertainty in $w$ and $\Omega_{m}$ dramatically increases the area of the $\sigma_{8}-\Omega_{m}$ plane allowed by the WMAP5 data. Moreover, we can see from Figure 14 that the WMAP constraint goes from being orthogonal to the cluster normalization condition to being parallel to it, implying that the cluster normalization condition cannot improve upon the dark energy constraints of WMAP alone. Indeed, a prior of the form $\sigma_{8}\left(\Omega_{m} / 0.25\right)^{0.41}=0.832 \pm 0.033$ has a minimal impact on the error bar in $w$. Fortunately, given this understanding, it is easy to see how to improve this situation: we need to introduce an additional observable which breaks the $w-\Omega_{m}$ degeneracy. As an example, in the above Figures we also show the $68 \%$ confidence intervals obtained for three additional analyses:

1. A joint WMAP5+BAO analysis, which includes the dark energy constraints derived by Eisenstein et al. (2005) using the Baryon Acoustic Oscillation (BAO) measurement from the SDSS LRG galaxy sample. 
TABLE 5

Cosmological Constraints From Multiple Cluster Abundance Experiments

\begin{tabular}{cccccc}
\hline \hline Source & Reference & $\gamma$ & $\sigma_{8}\left(\Omega_{m} / 0.25\right)^{\gamma}$ & $\sigma_{8}$ & $\Omega_{m}$ \\
\hline maxBCG Richness Function & this work & 0.41 & $0.832 \pm 0.033$ & $0.807 \pm 0.020$ & $0.270 \pm 0.019$ \\
X-ray Luminosity Function & Mantz et al. (2008) & 0.62 & $0.85 \pm 0.07$ & $0.809 \pm 0.028$ & $0.272 \pm 0.026$ \\
Temperature Function & Henry et al. (2008) & 0.30 & $0.80 \pm 0.04$ & $0.795 \pm 0.023$ & $0.258 \pm 0.025$ \\
Mass function estimated with $Y_{X}$ & Vikhlinin et al. (2008b) & 0.47 & $0.808 \pm 0.024$ & $0.798 \pm 0.017$ & $0.260 \pm 0.014$
\end{tabular}

Note. - The $\sigma_{8}$ and $\Omega_{m}$ constraint from the maxBCG + WMAP5 analysis quoted here differs very slightly from that presented in Figure 5 because of the simplified approach we have taken in this section for deriving the constraints (see text for details). Note Vikhlinin et al. (2008b) quote their result as $\sigma_{8}\left(\Omega_{m} / 0.25\right)^{0.47}=0.813 \pm 0.013($ stat $) \pm$ 0.02 (sys). For this study, we have simply added these two uncertainties in quadrature.
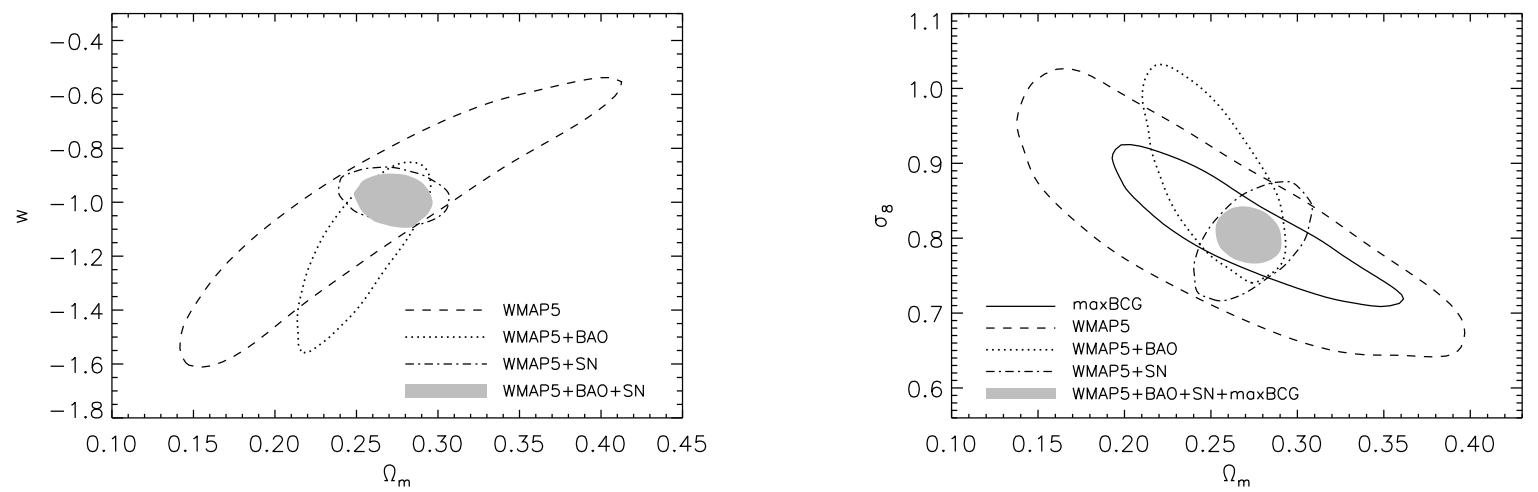

FIG. 14. - Parameter constraints on the $w-\Omega_{m}$ plane (left) and $\sigma_{8}-\Omega_{m}$ plane (right) in a flat $w$ CDM cosmology, for various data combinations. All contours shown are $68 \%$ confidence, and are obtained using the MCMC chain outputs downloaded from the LAMBDA website (http://lambda.gsfc.nasa.gov/). Despite the fact that the WMAP5 data constrain the amplitude of the primordial power spectrum with comparable accuracy in both a $\Lambda \mathrm{CDM}$ and $w \mathrm{CDM}$ cosmology, allowing $w$ to vary introduces a large degeneracy between $w$ and $\Omega_{m}$. This degeneracy severely degrades the WMAP constraints in the $\sigma_{8}-\Omega_{m}$ plane, as seen in the right panel. Adding new observables that break the $w-\Omega_{m}$ degeneracy restores the complementarity between WMAP5 and clusters in the $\sigma_{8}-\Omega_{m}$ plane, which helps improve dark energy constraints through the growth of structure.

2. A joint WMAP5+SN analysis, which draws on the Union combined dataset (Kowalski et al. 2008), a compilation of Super Nova (SN) data composed of the Riess et al. (2004, gold sample only), Astier et al. (2006), and Miknaitis et al. (2007) supernova samples.

3. A joint WMAP $5+\mathrm{BAO}+\mathrm{SN}$ analysis, which adds both BAO and SN measurements as extra observables.

In all cases, the confidence contours are estimated based on the MCMC data made publicly available by the WMAP team through the LAMBDA website (http://lambda.gsfc.nasa.gov/). These data sets break the $w-\Omega_{m}$ degeneracy from the WMAP5 data alone, and they restore the complementarity between WMAP5 and the cluster normalization condition in the $\sigma_{8}-\Omega_{m}$ plane, as illustrated in the lower panel of Figure 14.

Figure 15 shows the constraints in the $w-$ $\sigma_{8}\left(\Omega_{m} / 0.25\right)^{0.41}$ plane for the various analyses considered in Figure 14. The corresponding cosmological constraints are summarized in Table 6 . In order to compute how cluster abundances improve cosmological constraints, we have simply added the cluster normalization condition derived within the standard $\Lambda$ CDM cosmological model as a prior. While one might worry that letting the dark energy equation of state vary would degrade the uncertainty in the cluster normalization condition, in practice $w$ has a minimal impact provided one re-

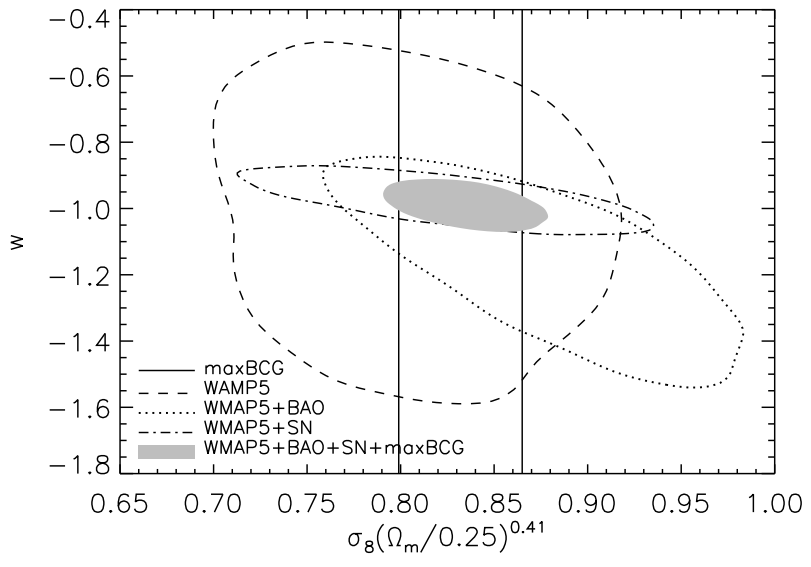

FIG. 15.- Confidence contours in the $w-\sigma_{8}\left(\Omega_{m} / 0.25\right)^{0.41}$ plane for analyses using various combinations of cosmological data. Contours all indicate $68 \%$ confidence. Provided the $w-\Omega_{m}$ degeneracy from Figure 14 is broken by an additional observable, cluster abundances can help constrain dark energy through the growth of structure between the time of last scattering and the low-redshift universe.

stricts oneself to low redshift cluster samples. For instance, varying $w$ by $\Delta w=0.1$ changes the comoving distance to the median redshift of maxBCG clusters by $\approx 1 \%$. The growth function is even less sensitive, varying by a mere $\approx 0.3 \%$. Thus, the cluster normalization condition from low-redshift cluster samples is essentially 
TABLE 6

Cosmological Constraints in a Flat $w$ CDM Cosmology

\begin{tabular}{cccc}
\hline \hline Experiment & $\Omega_{m}$ & $w$ & $\sigma_{8}$ \\
\hline WMAP5 & $0.266 \pm 0.086$ & $-1.05 \pm 0.34$ & $0.811 \pm 0.121$ \\
WMAP5+BAO & $0.251 \pm 0.027$ & $-1.20 \pm 0.24$ & $0.885 \pm 0.094$ \\
WMAP5+SN & $0.274 \pm 0.023$ & $-0.98 \pm 0.07$ & $0.798 \pm 0.053$ \\
WMAP5+maxBCG & $0.265 \pm 0.048$ & $-1.07 \pm 0.34$ & $0.815 \pm 0.061$ \\
WMAP5+SN+BAO & $0.274 \pm 0.015$ & $-0.995 \pm 0.067$ & $0.808 \pm 0.047$ \\
WMAP5+SN+maxBCG & $0.274 \pm 0.016$ & $-0.978 \pm 0.053$ & $0.801 \pm 0.026$ \\
WMAP5+BAO+maxBCG & $0.258 \pm 0.023$ & $-1.097 \pm 0.160$ & $0.831 \pm 0.044$ \\
WMAP5+BAO+SN+maxBCG & $0.272 \pm 0.013$ & $-0.989 \pm 0.053$ & $0.805 \pm 0.026$
\end{tabular}

Note. - The constraints quoted here are derived by multiplying the WMAP5 likelihoods with a Gaussian prior of our cluster normalization condition. We demonstrate in the text that results derived in this way are nearly identical to those from more detailed treatments.

independent of $w$.

To demonstrate this explicitly, we compare the results of Vikhlinin et al. (2008b) to the results from our simple analysis in which the cluster normalization condition $\sigma_{8}\left(\Omega_{m} / 0.25\right)^{0.47}=0.808 \pm 0.024$ from Vikhlinin et al. (2008b) is added to the data sets mentioned above. For a joint WMAP+BAO+clusters analysis, they find $w=-0.97 \pm 0.12$ while our simple analysis results in $w=$ $-1.04 \pm 0.13$. Note that the error bars are nearly identical, while the central value for $w$ differ by only half a $\sigma$. This is still true after adding supernovae as an additional constraint, in which case they find $w=-0.991 \pm 0.045$ compared to our $w=-0.971 \pm 0.048$. A similar conclusion can be reached for the Mantz et al. (2008) analysis. For a joint WMAP+supernovae $+f_{\text {gas }}+$ cluster abundance analysis, the find $w=-1.02 \pm 0.06$. With our simple analysis, and ignoring $f_{\text {gas }}$, we obtain $w=$ $-0.98 \pm 0.06$. This demonstrates that, to high accuracy, current cluster catalogs improve cosmological constraints on dark energy only through the low-redshift cluster normalization condition.

In summary, we have shown that cluster abundances help constrain the dark energy equation of state principally through the cluster normalization condition at low redshifts, which constrains the growth of structure between the epoch of recombination and today. However, the intrinsic degeneracy between $w$ and $\Omega_{m}$ given CMB data renders this test ineffective unless the degeneracy is broken by an additional data set. It is also worth remarking here that if we compare the results of a WMAP $5+\mathrm{SN}$ analysis to those obtained after including the maxBCG cluster normalization condition, the constraint on the dark energy equation of state is only improved at the $25 \%$ level, going from $\Delta w=0.07$ to $\Delta w=0.054$. This reflects the fact that distance-redshift relationships tend to be more sensitive to $w$ than the growth of structure. Nevertheless, the good agreement between the WMAP $5+\mathrm{SN}$ constraints and the cluster normalization is far from trivial. Indeed, the WMAP5+SN likelihood contours in the $\sigma_{8}-\Omega_{m}$ plane assume general relativity, so the good agreement with our data indicates that we are not able to resolve any departures from Einstein's theory of gravity (see also Rapetti et al. 2008; Mortonson et al. 2009). While quantitative constraints on such deviations are model dependent, our final error on $\sigma_{8}$ allow us to unambiguously state that models for which the growth factor between last scattering and today differ from our best fit $\Lambda \mathrm{CDM}$ model by $\approx 6 \%$ can be ruled out at the $2 \sigma$ level.

\subsection{Prospects For Improvement}

It is worth considering to what extent we can expect the cosmological constraints from maxBCG to improve with further study. Given that the two principal sources of systematic uncertainty are the amplitude of the weak lensing mass calibration and the prior on the scatter of the mass-richness relation, we focus here on those two quantities. More specifically, we re-analyze our data using artificially tight priors on each of these parameters individually, as well as on both parameters simultaneously. The tight priors adopted for this exercise are $\beta=1.00 \pm 0.01$ for the mass bias parameter, and $\sigma_{M \mid N_{200}}=0.45 \pm 0.02$ for the scatter in mass at fixed richness. We find that the uncertainties in $S_{8}=\sigma_{8}\left(\Omega_{m} / 0.25\right)^{0.41}$ for each of these analyses are:

1. fiducial analysis: $\Delta S_{8}=0.033$,

2. tight scatter prior alone: $\Delta S_{8}=0.026$,

3. tight mass bias prior alone: $\Delta S_{8}=0.025$, and

4. tight mass bias and scatter prior: $\Delta S_{8}=0.018$.

Thus, a tight prior on either the mass bias or the scatter parameter improves the principal maxBCG cosmological constraint by $25 \%$. If both priors are tightened, the improvement is as high as $50 \%$. The corresponding constraint on $w$ for this most optimistic scenario, assuming a joint WMAP $5+\mathrm{BAO}+\mathrm{SN}+$ maxBCG analysis, would be $\Delta w=0.049$, which is only a $7 \%$ improvement relative to the current constraint.

Are such improvements feasible? In principle, yes. Improvement of the mass bias parameter is possible through a follow-up spectroscopic program aimed at calibrating the mean lensing critical surface density of the lenssource pairs used to estimate the mean cluster masses. Likewise, an extensive X-ray follow-up program could in principle constrain the scatter in the mass-richness relation to high accuracy. In practice, realizing such tight priors might be difficult. For instance, given the current scatter estimate $\sigma_{M \mid N_{200}}=0.45$, we require $\approx 400 \mathrm{X}$-ray follow ups to achieve an uncertainty of $\Delta \sigma_{M \mid N_{200}} \approx 0.02$. Such an extensive program seems unlikely to be feasible any time in the near future. What is needed, then is a way to significantly reduce the number of followup observations necessary to improve our cosmological constraints. Fortunately, the number of follow-up observations necessary to achieve such accuracy scales as the 
square of the scatter $\sigma_{M \mid N_{200}}$, so the best thing to do at this point is probably to focus on constructing new richness estimators that better correlate with halo mass (see e.g. Rozo et al. 2008b; Reyes et al. 2008).

Finally, we note that there remains additional information about the maxBCG clusters that has not yet been incorporated into our analysis. This includes galaxy velocity dispersion data (Becker et al. 2007), X-ray data (Rykoff et al. 2008, while X-ray data was used to place a constraint on the scatter in mass at fixed richness, we did not otherwise use the X-ray data in this analysis), and clustering information (Estrada et al. 2008). Including these additional probes of cluster mass should help further improve our cosmological constraints.

\section{SUMMARY}

We have performed a joint analysis of the abundance and weak lensing mass estimates of the maxBCG clusters detected using SDSS imaging data. In addition to this data, a prior on the scatter in the mass-richness relation derived from demanding consistency between the weak lensing and X-ray mass estimates of the clusters. Our cosmological constraints can be summarized as

$$
\sigma_{8}\left(\Omega_{m} / 0.25\right)^{0.41}=0.832 \pm 0.033,
$$

which is consistent with and complementary to the latest WMAP results. With a joint maxBCG and WMAP5 analysis we find

$$
\begin{aligned}
\sigma_{8} & =0.807 \pm 0.020 \\
\Omega_{m} & =0.265 \pm 0.016 .
\end{aligned}
$$

These results firmly establish optical cluster studies as a method for deriving precise cosmological constraints. Importantly, our results are in excellent agreement with and of comparable precision to X-ray derived cluster abundance constraints, clearly demonstrating the robustness of galaxy clusters as a tool of precision cosmology.

We have discussed how and why galaxy clusters can help constrain dark energy evolution, demonstrating that even in those data sets where the evolution of cluster abundance with redshift is clearly detected, constraints on the dark energy density and equation of state of a joint WMAP and cluster abundance analysis are dominated by the low redshift cluster normalization condition $\sigma_{8} \Omega_{m}^{\gamma}=$ constant. These joint constraints are driven by the growth of the matter fluctuations between the time of last scattering and the low redshift universe. Thus, while cluster abundances provide only moderate improvements to dark energy constraints derived from joint WMAP and supernovae analysis, we have argued that they provide an important consistency test of general relativity. More specifically, our constraint on $\sigma_{8}$ allows us to rule out at the $2 \sigma$ lavel any models for which the growth of structure between last scattering and today differs from that of our best fit $\Lambda$ CDM model by more than $\sim 6 \%$.
At this time, the dominant systematic uncertainty in our analysis is the uncertainty in the weak lensing mass scale due to scatter in photometric redshift estimates of the source galaxies. In addition, improvements to our understanding of the scatter of the mass-richness relation could help tighten our cosmological constraints. Followup observations can help in this regard, but the number of follow ups necessary to have a significant impact on our results is currently very large. Fortunately, reducing the scatter of the mass-richness relation (see e.g. Reyes et al. 2008; Rozo et al. 2008b) may help reduce the number of follow up observations necessary to achieve improved constraints.

We thank Hao-Yi Wu for a careful reading of the manuscript and helpful comments. ER was funded by the Center for Cosmology and Astro-Particle Physics at The Ohio State University and by NSF grant AST 0707985. RHW was supported in part by the U.S. Department of Energy under contract number DE-AC02-76SF00515 and by a Terman Fellowship at Stanford University. ESR would like to thank the TABASGO foundation. TAM was funded in part by the National Science Foundation under Grant number AST-0807304. This work is supported in part by U.S. Department of Energy under contract No. DE-AC02-98CH10886. AEE acknowledges support from NSF AST-0708150.

Funding for the SDSS and SDSS-II has been provided by the Alfred P. Sloan Foundation, the Participating Institutions, the National Science Foundation, the U.S. Department of Energy, the National Aeronautics and Space Administration, the Japanese Monbukagakusho, the Max Planck Society, and the Higher Education Funding Council for England. The SDSS Web Site is http://www.sdss.org/.

The SDSS is managed by the Astrophysical Research Consortium for the Participating Institutions. The Participating Institutions are the American Museum of Natural History, Astrophysical Institute Potsdam, University of Basel, University of Cambridge, Case Western Reserve University, University of Chicago, Drexel University, Fermilab, the Institute for Advanced Study, the Japan Participation Group, Johns Hopkins University, the Joint Institute for Nuclear Astrophysics, the Kavli Institute for Particle Astrophysics and Cosmology, the Korean Scientist Group, the Chinese Academy of Sciences (LAMOST), Los Alamos National Laboratory, the Max-Planck-Institute for Astronomy (MPIA), the MaxPlanck-Institute for Astrophysics (MPA), New Mexico State University, Ohio State University, University of Pittsburgh, University of Portsmouth, Princeton University, the United States Naval Observatory, and the University of Washington.

\section{REFERENCES}

Allen, S. W., Schmidt, R. W., Fabian, A. C., \& Ebeling, H. 2003, MNRAS, 342, 287
Astier, P., Guy, J., Regnault, N., Pain, R., Aubourg, E., Balam, D. Basa, S., Carlberg, R. G., Fabbro, S., Fouchez, D., Hook, I. M., Howell, D. A., Lafoux, H., Neill, J. D., Palanque-Delabrouille, N., Perrett, K., Pritchet, C. J., Rich, J., Sullivan, M., Taillet, R., Aldering, G., Antilogus, P., Arsenijevic, V., Balland, C., Baumont, S., Bronder, J., Courtois, H., Ellis, R. S., Filiol, M., Gonçalves, A. C., Goobar, A., Guide, D., Hardin, D., Lusset, V., Lidman, C., McMahon, R., Mouchet, M., Mourao, A., Perlmutter, S., Ripoche, P., Tao, C., \& Walton, N. 2006, A\&A, 447,31 
Bahcall, N. A. \& Bode, P. 2003, ApJ, 588, L1

Bahcall, N. A., Dong, F., Bode, P., Kim, R., Annis, J., McKay, T. A., Hansen, S., Schroeder, J., Gunn, J., Ostriker, J. P., Postman, M., Nichol, R. C., Miller, C., Goto, T., Brinkmann, J., Knapp, G. R., Lamb, D. O., Schneider, D. P., Vogeley, M. S., \& York, D. G. 2003, ApJ, 585, 182

Becker, M. R., McKay, T. A., Koester, B., Wechsler, R. H., Rozo, E., Evrard, A., Johnston, D., Sheldon, E., Annis, J., Lau, E., Nichol, R., \& Miller, C. 2007, ApJ, 669, 905

Bond, J. R., Cole, S., Efstathiou, G., \& Kaiser, N. 1991, ApJ, 379, 440

Borgani, S., Rosati, P., Tozzi, P., Stanford, S. A., Eisenhardt, P. R., Lidman, C., Holden, B., Della Ceca, R., Norman, C., \& Squires, G. 2001, ApJ, 561, 13

Burenin, R. A., Vikhlinin, A., Hornstrup, A., Ebeling, H., Quintana, H., \& Mescheryakov, A. 2007, ApJS, 172, 561

Cohn, J. D., Evrard, A. E., White, M., Croton, D., \& Ellingson, E. 2007, MNRAS, 382, 1738

Cunha, C. 2008, ArXiv e-prints

Dunkley, J., Bucher, M., Ferreira, P. G., Moodley, K., \& Skordis, C. 2005, MNRAS, 356, 925

Dunkley, J., Komatsu, E., Nolta, M. R., Spergel, D. N., Larson, D., Hinshaw, G., Page, L., Bennett, C. L., Gold, B., Jarosik, N., Weiland, J. L., Halpern, M., Hill, R. S., Kogut, A., Limon, M., Meyer, S. S., Tucker, G. S., Wollack, E., \& Wright, E. L. 2008, ArXiv e-prints, 803

Eisenstein, D. J. \& Hu, W. 1999, ApJ, 511, 5

Eisenstein, D. J., Zehavi, I., Hogg, D. W., Scoccimarro, R., Blanton, M. R., Nichol, R. C., Scranton, R., Seo, H.-J., Tegmark, M., Zheng, Z., Anderson, S. F., Annis, J., Bahcall, N., Brinkmann, J., Burles, S., Castander, F. J., Connolly, A., Csabai, I., Doi, M., Fukugita, M., Frieman, J. A., Glazebrook, K., Gunn, J. E., Hendry, J. S., Hennessy, G., Ivezić, Z., Kent, S., Knapp, G. R., Lin, H., Loh, Y.-S., Lupton, R. H., Margon, B., McKay, T. A., Meiksin, A., Munn, J. A., Pope, A., Richmond, M. W., Schlegel, D., Schneider, D. P., Shimasaku, K., Stoughton, C., Strauss, M. A., SubbaRao, M., Szalay, A. S., Szapudi, I., Tucker, D. L., Yanny, B., \& York, D. G. 2005, ApJ, 633, 560

Estrada, J., Sefusatti, E., \& Frieman, J. A. 2008, ArXiv e-prints

Gladders, M. D., Yee, H. K. C., Majumdar, S., Barrientos, L. F., Hoekstra, H., Hall, P. B., \& Infante, L. 2007, ApJ, 655, 128

Henry, J. P. 2000, ApJ, 534, 565

-. 2004, ApJ, 609, 603

Henry, J. P. \& Arnaud, K. A. 1991, ApJ, 372, 410

Henry, J. P., Evrard, A. E., Hoekstra, H., Babul, A., \& Mahdavi, A. 2008, ArXiv e-prints

Hu, W. 2003, Phys. Rev. D, 67, 081304

Hu, W. \& Kravtsov, A. V. 2003, ApJ, 584, 702

Jenkins, A., Frenk, C. S., White, S. D. M., Colberg, J. M., Cole, S., Evrard, A. E., Couchman, H. M. P., \& Yoshida, N. 2001 MNRAS, 321, 372

Johnston, D. E., Sheldon, E. S., Tasitsiomi, A., Frieman, J. A., Wechsler, R. H., \& McKay, T. A. 2007a, ApJ, 656, 27

Johnston, D. E., Sheldon, E. S., Wechsler, R. H., Rozo, E., Koester, B. P., Frieman, J. A., McKay, T. A., Evrard, A. E., Becker, M. R., \& Annis, J. 2007b, ArXiv e-prints, 709

Koester, B. P., McKay, T. A., Annis, J., Wechsler, R. H., Evrard, A., Bleem, L., Becker, M., Johnston, D., Sheldon, E., Nichol, R., Miller, C., Scranton, R., Bahcall, N., Barentine, J., Brewington, H., Brinkmann, J., Harvanek, M., Kleinman, S., Krzesinski, J., Long, D., Nitta, A., Schneider, D. P., Sneddin, S., Voges, W., \& York, D. 2007a, ApJ, 660, 239

Koester, B. P., McKay, T. A., Annis, J., Wechsler, R. H., Evrard, A. E., Rozo, E., Bleem, L., Sheldon, E. S., \& Johnston, D. 2007b, ApJ, 660, 221
Kowalski, M., Rubin, D., Aldering, G., Agostinho, R. J., Amadon, A., Amanullah, R., Balland, C., Barbary, K., Blanc, G., Challis, P. J., Conley, A., Connolly, N. V., Covarrubias, R., Dawson, K. S., Deustua, S. E., Ellis, R., Fabbro, S., Fadeyev, V., Fan, X., Farris, B., Folatelli, G., Frye, B. L., Garavini, G., Gates, E. L., Germany, L., Goldhaber, G., Goldman, B., Goobar, A., Groom, D. E., Haissinski, J., Hardin, D., Hook, I., Kent, S., Kim, A. G., Knop, R. A., Lidman, C., Linder, E. V., Mendez, J., Meyers, J., Miller, G. J., Moniez, M., Mourão, A. M., Newberg, H., Nobili, S., Nugent, P. E., Pain, R., Perdereau, O., Perlmutter, S., Phillips, M. M., Prasad, V., Quimby, R., Regnault, N., Rich, J., Rubenstein, E. P., Ruiz-Lapuente, P., Santos, F. D., Schaefer, B. E., Schommer, R. A., Smith, R. C., Soderberg, A. M., Spadafora, A. L., Strolger, L.-G., Strovink, M., Suntzeff, N. B., Suzuki, N., Thomas, R. C., Walton, N. A., Wang, L., Wood-Vasey, W. M., \& Yun, J. L. 2008, ApJ, 686, 749

Kravtsov, A. V., Vikhlinin, A., \& Nagai, D. 2006, ApJ, 650, 128

Lima, M. \& Hu, W. 2004, Phys. Rev. D, 70, 043504

-. 2005, Phys. Rev. D, 72, 043006

Majumdar, S. \& Mohr, J. J. 2004, ApJ, 613, 41

Mandelbaum, R., Seljak, U., \& Hirata, C. M. 2008a, ArXiv e-prints, 805

Mandelbaum, R., Seljak, U., Hirata, C. M., Bardelli, S., Bolzonella, M., Bongiorno, A., Carollo, M., Contini, T., Cunha, C. E., Garilli, B., Iovino, A., Kampczyk, P., Kneib, J.-P., Knobel, C., Koo, D. C., Lamareille, F., Le Fèvre, O., Leborgne, J.-F., Lilly, S. J., Maier, C., Mainieri, V., Mignoli, M., Newman, J. A., Oesch, P. A., Perez-Montero, E., Ricciardelli, E., Scodeggio, M., Silverman, J., \& Tasca, L. 2008b, MNRAS, 386, 781

Mantz, A., Allen, S. W., Ebeling, H., \& Rapetti, D. 2008, MNRAS, 387,1179

Miknaitis, G., Pignata, G., Rest, A., Wood-Vasey, W. M., Blondin, S., Challis, P., Smith, R. C., Stubbs, C. W., Suntzeff, N. B., Foley, R. J., Matheson, T., Tonry, J. L., Aguilera, C., Blackman, J. W., Becker, A. C., Clocchiatti, A., Covarrubias, R., Davis, T. M., Filippenko, A. V., Garg, A., Garnavich, P. M., Hicken, M., Jha, S., Krisciunas, K., Kirshner, R. P., Leibundgut, B., Li, W., Miceli, A., Narayan, G., Prieto, J. L., Riess, A. G., Salvo, M. E., Schmidt, B. P., Sollerman, J., Spyromilio, J., \& Zenteno, A. 2007, ApJ, 666, 674

Mortonson, M. J., Hu, W., \& Huterer, D. 2009, Phys. Rev. D, 79, 023004

Pierpaoli, E., Scott, D., \& White, M. 2001, MNRAS, 325, 77

Press, W. H. \& Schechter, P. 1974, ApJ, 187, 425

Rapetti, D., Allen, S. W., Mantz, A., \& Ebeling, H. 2008, ArXiv e-prints

Reyes, R., Mandelbaum, R., Hirata, C. M., Bahcall, N., \& Seljak, U. 2008, ArXiv e-prints, 802

Riess, A. G., Strolger, L.-G., Tonry, J., Casertano, S., Ferguson, H. C., Mobasher, B., Challis, P., Filippenko, A. V., Jha, S., Li, W., Chornock, R., Kirshner, R. P., Leibundgut, B., Dickinson, M., Livio, M., Giavalisco, M., Steidel, C. C., Benítez, T., \& Tsvetanov, Z. 2004, ApJ, 607, 665

Rines, K., Diaferio, A., \& Natarajan, P. 2007, ApJ, 657, 183

Robertson, B., Kravtsov, A., Tinker, J., \& Zentner, A. 2008, ArXiv e-prints

Rozo, E., Dodelson, S., \& Frieman, J. A. 2004, Phys. Rev. D, 70, 083008

Rozo, E., Rykoff, E. S., Evrard, A., Becker, M., McKay, T., Wechsler, R. H., Koester, B. P., Hao, J., Hansen, S., Sheldon, E., Johnston, D., Annis, J., \& Frieman, J. 2008a, ArXiv e-prints arXiv: 0809.2794

Rozo, E., Rykoff, E. S., Koester, B. P., McKay, T., Hao, J., Evrard, A., Wechsler, R. H., Hansen, S., Sheldon, E., Johnston, D., Becker, M., Annis, J., Bleem, L., \& Scranton, R. 2008b, ArXiv e-prints

Rozo, E., Wechsler, R. H., Koester, B. P., Evrard, A. E., \& McKay, T. A. 2007a, ArXiv Astrophysics e-prints, astro-ph/0703574

Rozo, E., Wechsler, R. H., Koester, B. P., McKay, T. A., Evrard, A. E., Johnston, D., Sheldon, E. S., Annis, J., \& Frieman, J. A. 2007b, ArXiv Astrophysics e-prints, astro-ph/0703571.

Rykoff, E. S., McKay, T. A., Becker, M. R., Evrard, A., Johnston, D. E., Koester, B. P., Rozo, E., Sheldon, E. S., \& Wechsler, R. H. 2008, ApJ, 675, 1106

Schuecker, P., Böhringer, H., Collins, C. A., \& Guzzo, L. 2003, A\&A, 398, 867

Seljak, U. 2002, MNRAS, 337, 769 
Sheldon, E. S., Johnston, D. E., Scranton, R., Koester, B. P., McKay, T. A., Oyaizu, H., Cunha, C., Lima, M., Lin, H., Frieman, J. A., Wechsler, R. H., Annis, J., Mandelbaum, R., Bahcall, N. A., \& Fukugita, M. 2007, ArXiv e-prints, 709

Sheth, R. K. \& Tormen, G. 2002, MNRAS, 329, 61

Stanek, R., Rudd, D., \& Evrard, A. E. 2009, MNRAS, L172+

Tinker, J., Kravtsov, A. V., Klypin, A., Abazajian, K., Warren, M., Yepes, G., Gottlöber, S., \& Holz, D. E. 2008, ApJ, 688, 709

Viana, P. T. P. \& Liddle, A. R. 1996, MNRAS, 281, 323

-. 1999, MNRAS, 303, 535

Viana, P. T. P., Nichol, R. C., \& Liddle, A. R. 2002, ApJ, 569, L75

Vikhlinin, A., Burenin, R. A., Ebeling, H., Forman, W. R., Hornstrup, A., Jones, C., Kravtsov, A. V., Murray, S. S., Nagai, D., Quintana, H., \& Voevodkin, A. 2008a, ArXiv e-prints, 805
Vikhlinin, A., Kravtsov, A. V., Burenin, R. A., Ebeling, H., Forman, W. R., Hornstrup, A., Jones, C., Murray, S. S., Nagai, D., Quintana, H., \& Voevodkin, A. 2008b, ArXiv e-prints Voevodkin, A. \& Vikhlinin, A. 2004, ApJ, 601, 610

Warren, M. S., Abazajian, K., Holz, D. E., \& Teodoro, L. 2006, ApJ, 646, 881

White, S. D. M., Efstathiou, G., \& Frenk, C. S. 1993, MNRAS, 262,1023 